\title{
GENERALIZATIONS OF THE STACKED BASES THEOREM
}

\author{
PAUL HILL AND CHARLES MEGIBBEN
}

\begin{abstract}
Let $H$ be a subgroup of the free abelian group $G$. In order for there to exist a basis $\left\{x_{i}\right\}_{i \in I}$ of $G$ for which $H=\bigoplus_{i \in I}\left\langle n_{i} x_{i}\right\rangle$ for suitable nonnegative integers $n_{i}$, it is obviously necessary for $G / H$ to be a direct sum of cyclic groups. In the 1950's, Kaplansky raised the question of whether this condition on $G / H$ is sufficient for the existence of such a basis. J. Cohen and H. Gluck demonstrated in 1970 that the answer is "yes"; their result is known as the stacked bases theorem, and it extends the classical and well-known invariant factor theorem for finitely generated abelian groups. In this paper, we develop a theory that contains and, in fact, generalizes in several directions the stacked bases theorem. Our work includes a complete classification, using numerical invariants, of the various free resolutions of any abelian group.
\end{abstract}

\section{INTRODUCTION}

Let $A$ be an arbitrary additively written abelian group, and consider two free resolutions

$$
0 \rightarrow H \rightarrow G \rightarrow A \rightarrow 0
$$

$$
0 \rightarrow H^{\prime} \rightarrow G^{\prime} \rightarrow A \rightarrow 0
$$

where $H \rightarrow G$ and $H^{\prime} \rightarrow G^{\prime}$ denote the inclusion maps of $H$ and $H^{\prime}$, respectively, in the free abelian groups $G$ and $G$. We say that (1) and $\left(1^{\prime}\right)$ are equivalent provided there exists an isomorphism $\pi: G \rightarrow G^{\prime}$ such that $\pi(H)=H^{\prime}$. The problem of giving necessary and sufficient conditions for the free resolutions (1) and $\left(1^{\prime}\right)$ to be equivalent has gone unsolved now for nearly thirty years (see Problem 36 in [4]). Indeed, heretofore, there seems to have been no real progress on this problem beyond a solution in the special case where $A$ is assumed to be torsion free [3] and the classically known facts covering the case where $A$ is finitely generated. Furthermore, these two special cases provide no insight into the general solution. For example, in the first instance, the obvious necessary condition $\operatorname{rank}(H)=\operatorname{rank}\left(H^{\prime}\right)$ actually suffices for the equivalence of ( 1$)$ and $\left(1^{\prime}\right)$. The general solution for arbitrary $A$ involves the introduction of a countable number of cardinal invariants associated with the free resolution

Received by the editors November 16, 1987.

1980 Mathematics Subject Classification (1985 Revision). Primary 20 K99.

The authors were supported in part, respectively, by NSF grants DMS-8521770 and DMS8420900 . 
(1). If $p$ is a prime and if $C$ is a $p$-bounded abelian group (that is, $p C=0$ ), then $\operatorname{dim}(C)$ will denote the dimension, possibly transfinite, of $C$ as a vector space over the field $Z / p Z$. The new invariants required to resolve the equivalence problem are the cardinal numbers $\delta(p)=\operatorname{dim}\left(\frac{H+p G}{p G}\right)$ where $p$ ranges over the set of all rational primes.

Theorem 1.1. The free resolutions (1) and $\left(1^{\prime}\right)$ of the abelian group $A$ are equivalent if and only if, for each prime $p$,

$$
\operatorname{dim}\left(\frac{H+p G}{p G}\right)=\operatorname{dim}\left(\frac{H^{\prime}+p G^{\prime}}{p G^{\prime}}\right) .
$$

That condition (2) is necessary for the equivalence of $(1)$ and $\left(1^{\prime}\right)$ is, of course, trivial. The next section will be devoted to the proof that (2) does indeed imply (1) and $\left(1^{\prime}\right)$ are equivalent. We shall first, however, consider some of the simpler consequences of Theorem 1.1.

Corollary 1.2. If $A$ is an infinite abelian group and if both $\operatorname{rank}(G)$ and $\operatorname{rank}(G)$ are strictly greater than $|A|$, Then (1) and $\left(1^{\prime}\right)$ are equivalent if and only if $\operatorname{rank}(H)=\operatorname{rank}\left(H^{\prime}\right)$.

Proof. In general, $\operatorname{rank}(G)=\operatorname{dim}(G / p G)$ and $A / p A \cong G /(H+p G)$. Therefore we have

$$
\operatorname{rank}(G)=\operatorname{dim}\left(\frac{H+p G}{p G}\right)+\operatorname{dim}(A / p A) .
$$

In case $\operatorname{rank}(G)>|\dot{A}| \geq \aleph_{0}$, we clearly have $\operatorname{rank}(H)=\operatorname{rank}(G)>\operatorname{dim}(A / p A)$. Thus, $\operatorname{dim}\left(\frac{H+p G}{p G}\right)=\operatorname{rank}(H)$ for all primes $p$, and the corollary follows immediately from Theorem 1.1 .

For an infinite group $A$, Corollary 1.2 makes it clear that it is only when $\operatorname{rank}(G)=|A|$ that our new invariants are required. But $\operatorname{rank}(G)=|A|$ tends to be the rule, not the exception; see Corollary 1.3. Even though our next corollary can be established by an elementary argument, the short proof we give below serves as a nice example of a dominant theme in the theory of abelian groups, namely, the interplay between uniqueness and existence theorems. The uniqueness theorem here is Theorem 1.1.

Corollary 1.3. If $\operatorname{rank}(G)>|A| \geq \aleph_{0}$ in the free resolution (1), then there exists a direct decomposition $G=G_{1} \oplus G_{2}$ with $\operatorname{rank}\left(G_{1}\right)=|A|$ and $G_{2} \subseteq H$.

Proof. Certainly there is a free group $G_{1}^{\prime}$, with $\operatorname{rank}\left(G_{1}^{\prime}\right)=|A|$, and $G_{1}^{\prime} / H_{1}^{\prime} \cong A$ for some subgroup $H_{1}^{\prime}$. Now take $G=G_{1}^{\prime} \oplus F$ and $H^{\prime}=H_{1}^{\prime} \oplus F$ where $F \cong G$. Then if $\left(1^{\prime}\right)$ is the free resolution of $A$ arising from the particular choice of $G$ and $H^{\prime}$, Corollary 1.2 tells us that $(1)$ and $\left(1^{\prime}\right)$ are equivalent since clearly $\operatorname{rank}\left(H^{\prime}\right)=\operatorname{rank}(F)=\operatorname{rank}(H)$. Finally, take $G_{1}=\pi^{-1}\left(G_{1}^{\prime}\right)$ and $G_{2}=\pi^{-1}(F)$ where $\pi$ is any isomorphism of $G$ onto $G$ with $\pi(H)=H^{\prime}$.

Our next result includes Erdös' theorem [3] and illustrates the effect the structure of $A$ can have on the permissible values of the invariants $\operatorname{dim}\left(\frac{H+p G}{p G}\right)$. 
Corollary 1.4. If $A$ is torsion free or divisible, then (1) and $\left(1^{\prime}\right)$ are equivalent if and only if $\operatorname{rank}(H)=\operatorname{rank}\left(H^{\prime}\right)$.

Proof. If $A$ is torsion free, then $H \cap p G=p H$ and $\operatorname{dim}\left(\frac{H+p G}{p G}\right)=\operatorname{dim}(H / p H)$ $=\operatorname{rank}(H)$ for all primes $p$. If $A$ is divisible, then $H+p G=G$ and $\operatorname{dim}\left(\frac{H+p G}{p G}\right)=\operatorname{dim}(G / p G)=\operatorname{rank}(G)$. But since, in general,

$$
\operatorname{rank}(G) \geq \operatorname{rank}(H)=\operatorname{dim}(H / p H) \geq \operatorname{dim}\left(\frac{H}{H \cap p G}\right)=\operatorname{dim}\left(\frac{H+p G}{p G}\right),
$$

we also have $\operatorname{dim}\left(\frac{H+p G}{p G}\right)=\operatorname{rank}(H)$ for all primes $p$ when $A \cong G / H$ is divisible.

The following striking but simple consequence of Theorem 1.1 was apparently unknown heretofore.

Corollary 1.5. If $A$ is a nonzero divisible group in (1), then $H$ contains an infinite rank direct summand of $G$.

Proof. Since $A$ is infinite and contains no nontrivial free direct summand, $\operatorname{rank}(H)=\operatorname{rank}(G) \geq \aleph_{0}$. Let $\left(1^{\prime}\right)$ be the free resolution of $A$ that arises from taking $G^{\prime}=G \oplus G$ and $H^{\prime}=G \oplus H$. Then Corollary 1.4 implies that (1) and $\left(1^{\prime}\right)$ are equivalent. Therefore, just as in the proof of Corollary 1.3, we can exploit the equivalence of $(1)$ and $\left(1^{\prime}\right)$ to argue that $H$ contains a direct summand $G_{1}$ of $G$ with $\operatorname{rank}\left(G_{1}\right)=\operatorname{rank}(G)$.

There are, of course, other instances where the structure of $A$ is such that one is led to the same conclusion as in Corollary 1.4 regarding the equivalence of free resolutions. As one further such example, we offer the following result.

Corollary 1.6. If $A$ is a primary abelian group containing a basic subgroup $B$ with $|B|<|A|$, then the free resolutions (1) and $\left(1^{\prime}\right)$ are equivalent if and only if $\operatorname{rank}(H)=\operatorname{rank}\left(H^{\prime}\right)$.

Proof. Since $A$ is infinite and contains no nontrivial free direct summand, we must have $\operatorname{rank}(H)=\operatorname{rank}(G) \geq \aleph_{0}$ in (1). If $p$ is not the relevant prime for the primary group $A$, then $\operatorname{dim}(A / p A)=0$ and therefore equation (3) above yields $\operatorname{rank}(H)=\operatorname{rank}(G)=\operatorname{dim}\left(\frac{H+p G}{p G}\right)$. On the other hand, if $A$ is $p$-primary then $\operatorname{dim}(A / p A)=\operatorname{dim}(B / p B)<|A| \leq \operatorname{rank}(G)$, and (3) once again yields $\operatorname{dim}\left(\frac{H+p G}{p G}\right)=\operatorname{rank}(H)$. In summary, $\operatorname{dim}\left(\frac{H+p G}{p G}\right)=\operatorname{rank}(H)$ for all primes $p$ and thus the corollary follows from Theorem 1.1.

All the preceding corollaries notwithstanding, there certainly exist many groups $A$ for which the condition $\operatorname{rank}(H)=\operatorname{rank}\left(H^{\prime}\right)$ does not suffice for (1) and $\left(1^{\prime}\right)$ to be equivalent. In fact, once our new invariants have been identified, the necessity of their preservation in Theorem 1.1 already serves as adequate inspiration for the construction of an example that defeats a previously suggested sufficient condition for the equivalence of free resolutions (see Problem 36 in [4]). This example appears in the proof of our final corollary in this section to Theorem 1.1. 
Corollary 1.7. Let $\operatorname{rank}(H)=\operatorname{rank}\left(H^{\prime}\right)$ in (1) and $\left(1^{\prime}\right)$, and suppose furthermore that the following condition is satisfied: If $H$ contains a direct summand $K$ of $G$, then $H^{\prime}$ contains a direct summand $K^{\prime}$ of $G^{\prime}$ with $\operatorname{rank}\left(K^{\prime}\right)=\operatorname{rank}(K)$, and vice versa. Then (1) and ( $\left.1^{\prime}\right)$ need not necessarily be equivalent.

Proof. Let $G=G_{1} \oplus G_{2}$ where both $G_{1}$ and $G_{2}$ are free groups of rank $\aleph_{0}$, and take $H=6 G_{1} \oplus 10 G_{2}$. Next let $G$ be another free group of rank $\aleph_{0}$ and choose $H^{\prime}=30 G^{\prime}$. A quick consideration of the primary components leads to the conclusion that $G / H \cong G^{\prime} / H^{\prime}$. Since $H \subseteq 2 G$ and $H^{\prime} \subseteq 2 G^{\prime}$, neither $H$ nor $H^{\prime}$ can contain a nontrivial direct summand of $G$ or $G^{\prime}$, respectively. Thus the condition in 1.7 relating to the containment of direct summands of $G$ and $G$ is satisfied vacuously. Nevertheless, the corresponding free resolutions (1) and $\left(1^{\prime}\right)$ are not equivalent. Indeed, for $p=3, \operatorname{dim}\left(\frac{H+p G}{p G}\right)=\aleph_{0}$ and $\operatorname{dim}\left(\frac{H^{\prime}+p G^{\prime}}{p G^{\prime}}\right)=0$.

As promised above, we shall prove Theorem 1.1 in $\S 2$. In the third section, we shall use this theorem to give a new proof of the stacked bases theorem [2]. We therefore can interpret the stacked bases theorem as a special case of our theory and can now view it from a more general perspective. In $\S 4$, we provide an existence theorem delineating precisely the permissible values of the invariants $\delta(p)=\operatorname{dim}\left(\frac{H+p G}{p G}\right)$ in terms of the structure of the group $A \cong G / H$. Thus, using numerical invariants, we classify the free resolutions of any abelian group $A$. One striking consequence of this existence theorem is that if $A$ is a countable reduced torsion group with infinitely many unbounded primary components, then $A$ possesses $2^{\aleph_{0}}$ different free resolutions (1) with $\operatorname{rank}(H)=\operatorname{rank}(G)=\aleph_{0}$. Finally, in $\S 5$ we consider the problem of lifting direct decompositions of the group $A$ to corresponding decompositions of a given free resolution of $A$ and, in particular, establish a theorem for all infinite cardinals $m$ that reduces to the stacked bases theorem when $m=\aleph_{0}$.

\section{THE EQUiVALENCE THEOREM}

We begin the proof of Theorem 1.1 by stating what we might call the classical version of the stacked bases theorem (or, for those who prefer the more traditional terminology, an extended version of the invariant factor theorem).

Proposition 2.1. Let $H$ be a subgroup of the finite rank free group $G$. Then there exists a basis $x_{1}, \ldots, x_{m}$ for $G$ and a finite sequence of nonnegative integers $n_{1}, \ldots, n_{m}$ such that $n_{i} \mid n_{i+1}$ for $i=1,2, \ldots, m-1$ and $H$ is generated by $n_{1} x_{1}, \ldots, n_{m} x_{m}$. Moreover, the $n_{i}$ 's are uniquely determined by the rank of $H$ and the structure of the finitely generated group $A=G / H$.

Proof. For the existence of the $x_{i}$ 's and $n_{i}$ 's, see Lemma 15.4 in [5]. Let $l$ and $k$ be, respectively, the largest integers such that $n_{l}=1$ and $n_{k} \neq 0$. Then $k=\operatorname{rank}(H)$ since $n_{1} x_{1}, \ldots, n_{k} x_{k}$ form a basis for $H$, and $m-k$ is just the torsion free rank of $A=G / H$. If $A_{t}$ denotes the torsion subgroup of $A$, then $A_{t}=\left\langle a_{1}\right\rangle \oplus \cdots \oplus\left\langle a_{s}\right\rangle$ where $s=k-l$ and $O\left(a_{i}\right) \mid O\left(a_{i+1}\right)$ for $i=1, \ldots, s-1$ 
since $O\left(a_{i}\right)=n_{l+i}$. But in any such direct decomposition of a finite group, the $O\left(a_{i}\right)$ 's are uniquely determined (see, for example, Exercise 12 on p. 82 of [5]). From these observations, the final assertion of the proposition is evident.

Our next preliminary result will also play an important role in $\S 4$ where we determine the permissible values of the invariants $\operatorname{dim}\left(\frac{H+p G}{p G}\right)$ in terms of the structure of the quotient group $G / H$.

Proposition 2.2. If $H$ is a subgroup of the free group $G$ and if $S$ is an infinite set of primes, then

$$
\sup _{p \in S}\left(\operatorname{dim}\left(\frac{H+p G}{p G}\right)\right)=\operatorname{rank}(H) .
$$

Proof. For each prime $p$, let $\delta(p)=\operatorname{dim}\left(\frac{H+p G}{p G}\right)$. Suppose that for an infinite set $S$ of primes the alternative $\sup _{p \in S} \delta(p)<\operatorname{rank}(H)$ holds. In view of Proposition 2.1, it is clear that $H$ cannot have finite rank because in that case there exists a $p \in S$ not relevant to $G / H$ and consequently $(H+p G) / p G \cong$ $H /(H \cap p G)=H / p H$. Thus $\delta(p)$ would be equal to $\operatorname{rank}(H)$.

Actually, we can conclude even more from the preceding observation. Suppose that $\operatorname{rank}(H)$ is infinite. Given any finite rank summand $G_{1}$ of $G$, there exists a prime $p \in S$ for which

$$
\operatorname{dim}\left(\frac{\left(H \cap G_{1}\right)+p G_{1}}{p G_{1}}\right)=\operatorname{rank}\left(H \cap G_{1}\right) .
$$

But the mapping $\left(\left(H \cap G_{1}\right)+p G_{1}\right) / p G_{1} \rightarrow(H+p G) / p G$ is monic, so $\sup _{p \in S} \delta(p)$ must be infinite since there is no fixed bound on $\operatorname{rank}\left(H \cap G_{1}\right)$ when $G_{1}$ is thought of as a variable finite rank summand of $G$. We have concluded that $\sup _{p \in S} \delta(p)=\operatorname{rank}(H)$ if $\operatorname{rank}(H) \leq \aleph_{0}$.

Finally, assume that $\operatorname{rank}(H)>\aleph_{0}$. If $\sup _{p \in S} \delta(p)<\operatorname{rank}(H)$, it is clear that $G=G_{1} \oplus G_{2}$ where

$$
\operatorname{rank}\left(G_{1}\right) \leq \aleph_{0} \sup _{p \in S} \delta(p)<\operatorname{rank}(H)
$$

and $H \subseteq G_{1}+p G$ for all $p \in S$. This, however, is absurd since there must exist an element $h=g_{1}+g_{2}$ in $H$ with $g_{1}$ in $G_{1}$ and $g_{2} \neq 0$ in $G_{2}$, but the preceding condition requires that $g_{2} \in p G$ for all $p \in S$.

Corollary 2.3. If $H$ and $H^{\prime}$ are subgroups of the free groups $G$ and $G$, respectively, such that

$$
\operatorname{dim}\left(\frac{H+p G}{p G}\right)=\operatorname{dim}\left(\frac{H^{\prime}+p G^{\prime}}{p G^{\prime}}\right) \text { for all primes } p
$$

and

$$
G / H \cong G^{\prime} / H^{\prime}
$$

then $\operatorname{rank}(H)=\operatorname{rank}\left(H^{\prime}\right)$ and $\operatorname{rank}(G)=\operatorname{rank}\left(G^{\prime}\right)$.

Proof. By Proposition 2.2, condition (1) implies that $\operatorname{rank}(H)=\operatorname{rank}\left(H^{\prime}\right)$. In case $\operatorname{rank}(H)=\operatorname{rank}(G)$ and $\operatorname{rank}\left(H^{\prime}\right)=\operatorname{rank}\left(G^{\prime}\right)$, then the proof is complete. 
Suppose, however, that $\operatorname{rank}(H)<\operatorname{rank}(G)$. Then we have a direct decomposition $G=G_{1} \oplus G_{2}$ where $H \subseteq G_{1}$ and $\operatorname{rank}\left(G_{1}\right)=\operatorname{rank}(H)$. This observation is, of course, trivial when $\operatorname{rank}(H)$ is infinite and follows from Proposition 2.1 when $H$ has finite rank. Therefore $\operatorname{rank}(G)=\operatorname{rank}(H)+\operatorname{rank}\left(G_{2}\right)$ and $G / H$ contains a free direct summand having the same rank as $G_{2}$. But since $G / H \cong G / H^{\prime}$, the latter group also contains a free direct summand $K^{\prime} / H^{\prime}$ with $\operatorname{rank}\left(K^{\prime} / H^{\prime}\right)=\operatorname{rank}\left(G_{2}\right)$. Thus $K^{\prime}=H^{\prime} \oplus G_{2}^{\prime}$ where $G_{2}^{\prime} \cong G_{2}$ and consequently

$$
\operatorname{rank}\left(G^{\prime}\right) \geq \operatorname{rank}\left(K^{\prime}\right)=\operatorname{rank}\left(H^{\prime}\right) \oplus \operatorname{rank}\left(G_{2}^{\prime}\right)=\operatorname{rank}(G) .
$$

Hence we also have $\operatorname{rank}\left(G^{\prime}\right)>\operatorname{rank}(H)=\operatorname{rank}\left(H^{\prime}\right)$ and, by symmetry, $\operatorname{rank}(G)$ $\geq \operatorname{rank}\left(G^{\prime}\right)$. We conclude that $\operatorname{rank}(G)=\operatorname{rank}(G)$, and the proof is complete.

The proof of Theorem 1.1 in the special case where $H$ is finitely generated should now be more or less obvious. In view of Corollary 2.3 , the hypotheses of the theorem imply that $\operatorname{rank}(H)=\operatorname{rank}\left(H^{\prime}\right)$ and $\operatorname{rank}(G)=\operatorname{rank}\left(G^{\prime}\right)$. Thus we have direct decompositions $G=G_{1} \oplus G_{2}$ and $G^{\prime}=G_{1}^{\prime} \oplus G_{2}^{\prime}$ where $H \subseteq$ $G_{1}, H^{\prime} \subseteq G_{1}^{\prime}, \operatorname{rank}\left(G_{1}\right)=\operatorname{rank}(H)=\operatorname{rank}\left(H^{\prime}\right)=\operatorname{rank}\left(G_{1}^{\prime}\right)$ and $\operatorname{rank}\left(G_{2}\right)=$ $\operatorname{rank}\left(G_{2}^{\prime}\right)$. In particular, $G_{2} \cong G_{2}$. Moreover, by Proposition 2.1, we also have direct decompositions $G_{1}=\left\langle x_{1}\right\rangle \oplus \cdots \oplus\left\langle x_{m}\right\rangle$ and $G_{1}^{\prime}=\left\langle x_{1}^{\prime}\right\rangle \oplus \cdots \oplus\left\langle x_{m}^{\prime}\right\rangle$ where $H=\left\langle n_{1} x_{1}\right\rangle \oplus \cdots \oplus\left\langle n_{m} x_{m}\right\rangle$ and $H^{\prime}=\left\langle n_{1}^{\prime} x_{1}^{\prime}\right\rangle \oplus \cdots \oplus\left\langle n_{m}^{\prime} x_{m}^{\prime}\right\rangle$. Since $G_{1} / H$ and $G_{1}^{\prime} / H^{\prime}$ are just the maximal torsion subgroups of the isomorphic groups $G / H$ and $G^{\prime} / H^{\prime}$, respectively, we may assume that $n_{i}=n_{i}^{\prime}$ for all $i$. Therefore the desired isomorphism $\pi: G \rightarrow G$ is obtained by setting $\pi\left(x_{i}\right)=x_{i}^{\prime}$ and taking $\pi \mid G_{2}$ to be an arbitrary isomorphism of $G_{2}$ onto $G_{2}^{\prime}$.

In the case when $H$ is not finitely generated, there is surprisingly an even stronger version of Theorem 1.1. In fact, in this case, we shall show that the isomorphism $\pi: G \rightarrow G$ can be chosen to induce any given isomorphism $\phi: G / H \rightarrow G^{\prime} / H^{\prime}$ in the sense that $\phi(x+H)=\pi(x)+H^{\prime}$ for all $x \in G$. Clearly $\pi(H)=H^{\prime}$ if $\pi$ induces the isomorphism $\phi$. No such strengthening is available when $H$ has finite rank since, when $p$ is a prime, $Z / p Z$ has $p-1$ automorphisms and $Z$ has only two automorphisms.

Theorem 2.4. Let $\phi: G / H \rightarrow G / H^{\prime}$ be an isomorphism where $H$ and $H^{\prime}$ are infinite rank subgroups of the free groups $G$ and $G$, respectively. Then there is an isomorphism $\pi: G \rightarrow G$ inducing $\phi$ if and only if, for each prime $p$,

$$
\operatorname{dim}\left(\frac{H+p G}{p G}\right)=\operatorname{dim}\left(\frac{H^{\prime}+p G^{\prime}}{p G^{\prime}}\right) .
$$

Our proof of Theorem 2.4 will be accomplished through the following lemma.

Lemma 2.5. Assume the hypotheses of Theorem 2.4 and suppose $\pi: M \rightarrow M^{\prime}$ is an isomorphism with the following conditions satisfied:

(1) $M$ and $M^{\prime}$ are direct summands of $G$ and $G^{\prime}$, respectively.

(2) $\operatorname{rank}(M)<\operatorname{rank}(H)$ and $\operatorname{rank}\left(M^{\prime}\right)<\operatorname{rank}\left(H^{\prime}\right)$.

(3) $\phi(z+H)=\pi(z)+H^{\prime}$ for all $z \in M$. 
(4) For all primes $p$,

$$
\operatorname{dim}\left[\frac{H}{H \cap(M+p G)}\right]=\operatorname{dim}\left[\frac{H^{\prime}}{H^{\prime} \cap\left(M^{\prime}+p G^{\prime}\right)}\right] .
$$

Then if $S$ and $S^{\prime}$ are arbitrary finite subsets of $G$ and $G^{\prime}$, respectively, there is an isomorphism $\tau: N \rightarrow N^{\prime}$ extending $\pi$ such that $N / M$ and $N^{\prime} / M^{\prime}$ are both finitely generated, $S \subseteq N, S^{\prime} \subseteq N^{\prime}$ and conditions (1)-(4) continue to hold with $\tau, N$ and $N^{\prime}$ replacing $\pi, M$ and $M^{\prime}$, respectively.

Proof. Since the conditions (1)-(4) are symmetric with respect to $G$ and $G$ and since any finite subset of $G$ can be captured in a direct summand of the form $M \oplus\left\langle x_{1}\right\rangle \oplus \cdots \oplus\left\langle x_{n}\right\rangle$ with the $x_{i}$ 's selected from a basis of a complementary summand of $M$ (and similarly for a finite subset of $G$ ), it clearly suffices to prove the following: If $N=M \oplus\langle x\rangle$ is a direct summand of $G$, then there exists an isomorphism $\tau: N \rightarrow N^{\prime}$ extending $\pi$ with conditions (1)-(4) satisfied for $\tau, N$ and $N^{\prime}$. We shall repeatedly use the elementary fact that if $y \in G^{\prime}$, then $y=m y^{\prime}$ where $m$ is a positive integer and $\left\langle y^{\prime}\right\rangle$ is a direct summand of $G$. This, of course, follows from the well-known fact that if $y=t_{1} x_{1}+\cdots+t_{n} x_{n}$ where $\left(t_{1}, \ldots, t_{n}\right)=1$ and $\left\langle x_{1}\right\rangle \oplus \cdots \oplus\left\langle x_{n}\right\rangle$ is a direct summand of $G^{\prime}$, then $\langle y\rangle$ is also a direct summand. Another observation, the easy proof of which we leave to the reader, that we shall need frequently is the following: If $N=M \oplus\langle x\rangle$ is a direct summand of $G$ with $x \neq 0$,

$$
\operatorname{dim}\left[\frac{H \cap(N+p G)}{H \cap(M+p G)}\right]=1 \quad \text { if and only if } x \in H+M+p G .
$$

The proof of the existence of the desired $\tau$ and $N^{\prime}$ reduces to the consideration of two mutually exclusive cases.

Case I. $x \in M+H$. Since $M \oplus\langle x\rangle=M \oplus\langle x-z\rangle$ for any $z \in M$, we may assume without loss of generality that $x \in H$. Our plan of attack in this case is to show that there will exist an $x^{\prime} \in H^{\prime}$ such that $N^{\prime}=M^{\prime} \oplus\left\langle x^{\prime}\right\rangle$ is a direct summand of $G^{\prime}$. Once such an $x^{\prime}$ is found, we will take $\tau: N \rightarrow N^{\prime}$ to be that unique extension of $\pi$ with $\tau(x)=x^{\prime}$. Since $\phi(x+H)=0+H^{\prime}=$ $x^{\prime}+H^{\prime}=\tau(x)+H^{\prime}$, condition (3) will continue to hold for $\tau$. Clearly (2) will also remain intact for $N$ and $N^{\prime}$ since both $H$ and $H^{\prime}$ have infinite rank. Next note that, since $x \in H$ and $x^{\prime} \in H^{\prime},(*)$ and its analogue in $G^{\prime}$ imply that

$$
\operatorname{dim}\left[\frac{H \cap(N+p G)}{H \cap(M+p G)}\right]=1=\operatorname{dim}\left[\frac{H^{\prime} \cap\left(N^{\prime}+p G^{\prime}\right)}{H^{\prime} \cap\left(M^{\prime}+p G^{\prime}\right)}\right]
$$

for all primes $p$. From this observation, it clearly follows that (4) is satisfied by $N$ and $N^{\prime}$.

It remains, of course, to prove the existence of the desired $x^{\prime} \in H^{\prime}$. By (2), we at least know that there is an $x^{\prime} \in H^{\prime} \backslash M^{\prime}$. We can then write $x^{\prime}=z^{\prime}+m b$ where $z^{\prime} \in M^{\prime}, m$ is a positive integer and $G^{\prime}=M^{\prime} \oplus\langle b\rangle \oplus C$. We shall 
show that it is always possible to choose such an $x^{\prime}$ with $m=1$. To prove this, it suffices to demonstrate that, given such an $x^{\prime}$ with $m \neq 1$, we can then find an $x_{1}^{\prime}=z_{1}^{\prime}+m_{1} b_{1}$ in $H^{\prime}$ with $z_{1}^{\prime} \in M^{\prime}, G^{\prime}=M^{\prime} \oplus\left\langle b_{1}\right\rangle \oplus C_{1}$ and $m_{1}$ a proper positive factor of $m$. Assuming then that $m \neq 1$, let $p$ be any prime factor of $m$. Since $x \in H$ and $x \notin M+p G$, condition (4) implies that there exists an $h_{p}^{\prime} \in H^{\prime} \backslash\left(M^{\prime}+p G\right)$. We can then write $h_{p}^{\prime}=$ $z_{p}^{\prime}+n b+k c$ where $z_{p}^{\prime} \in M^{\prime}, n \in Z$ and $\langle c\rangle$ is a direct summand of $C$. Notice that $p$ cannot divide both $k$ and $n$ since $n b+k c \notin p G$. If $m \mid n$, say $n=m t$, then take $x_{1}^{\prime}=(1-t) x^{\prime}+h_{p}^{\prime}=z_{1}^{\prime}+m b+k c=z_{1}^{\prime}+m_{1} b_{1}$ where $z_{1}^{\prime}=(1-t) z^{\prime}+z_{p}^{\prime}$ is in $M^{\prime}, m_{1}=(m, k)$ is a proper factor of $m$ and $\left\langle b_{1}\right\rangle$ is a direct summand of $\langle b\rangle \oplus C$. If $m+n$, then $d=(m, n)$ is a proper factor of $m$ and we can write $d=\alpha m+\beta n$ for appropriate integers $\alpha$ and $\beta$. Now let $x_{1}^{\prime}=\alpha x^{\prime}+\beta h_{p}^{\prime}=z_{1}^{\prime}+d b+\beta k c=z_{1}^{\prime}+m_{1} b_{1}$ where $z_{1}^{\prime}=\alpha z^{\prime}+\beta z_{p}^{\prime}$ is in $M^{\prime}, m_{1}=(d, \beta k)$ is a proper factor of $m$ and $\left\langle b_{1}\right\rangle$ is a direct summand of $\langle b\rangle \oplus C$. We conclude that there does indeed exist an $x^{\prime}=z^{\prime}+b$ in $H^{\prime}$ such that $z^{\prime} \in M^{\prime}$ and $N^{\prime}=M^{\prime} \oplus\langle b\rangle$ is a direct summand of $G^{\prime}$. Finally, observe that $N^{\prime}=M^{\prime} \oplus\left\langle x^{\prime}\right\rangle$ and, consequently, the proof is complete for Case $\mathrm{I}$.

Case II. $x \notin M+H$. Choose any $w \in G$ such that $w+H^{\prime}=\phi(x+H)$. First observe that $w \notin M^{\prime}$, since otherwise there would exist a $z \in M$ such that $\pi(z)=w$ and then we would have the contradiction $x-z \in H$ because (3) would imply $\phi((x-z)+H)=0+H^{\prime}$. The strategy in this case is to show that we can find some appropriate $h^{\prime} \in H^{\prime}$ so that $x^{\prime}=w+h^{\prime}$ will have the property that $N^{\prime}=M^{\prime} \oplus\left\langle x^{\prime}\right\rangle$ is a direct summand of $G^{\prime}$. We begin by writing $w=z^{\prime}+m b$ where $z^{\prime} \in M^{\prime}, m$ is a positive integer and $G^{\prime}=M^{\prime} \oplus\langle b\rangle \oplus C$. Under these restraints, choose $m$ minimal. Because of (2), $H^{\prime} \cap C \neq 0$ and therefore we have a nonzero $h_{0}^{\prime}=t c$ in $H^{\prime}$ where $t$ is a positive integer and $C=\langle c\rangle \oplus D$. Consider the element $w+h_{0}^{\prime}=z^{\prime}+m b+t c$. We first observe that that $m \mid t$. Indeed if $m+t$, then $m_{1}=(m, t)$ is a proper factor of $m$ and $w+h_{0}^{\prime}=z^{\prime}+m_{1} b_{1}$ where $G^{\prime}=M^{\prime} \oplus\left\langle b_{1}\right\rangle \oplus C_{1}$, which is contrary to the choice of $m$. Thus, we do indeed have $w+h_{0}^{\prime}=z^{\prime}+m b+t c$ where $m \mid t$ and $G^{\prime}=M^{\prime} \oplus\langle b\rangle \oplus\langle c\rangle \oplus D$.

If $m=1$, then $w+h_{0}^{\prime}$ will be our desired $x^{\prime}$. Assuming that $m \neq 1$, we now introduce the finite set $P$ consisting of all primes $p$ such that $p \leq t$ and

$$
\operatorname{dim}\left[\frac{H^{\prime}}{H^{\prime} \cap\left(M^{\prime}+p G^{\prime}\right)}\right] \neq 0 .
$$

Since $\phi(x+H)=w+H^{\prime}$, observe that (*) combined with (3) and (4) yields the following sufficient condition for membership in $P$ :

$$
\text { If } p \leq t \text { and } w \in H^{\prime}+M^{\prime}+p G^{\prime} \text {, then } p \in P \text {. }
$$

In particular, since $m \mid t$, any prime factor of $m$ will be in $P$. Now choose, for each $p \in P$, an element $h_{p}^{\prime} \in H^{\prime} \backslash\left(M^{\prime}+p G^{\prime}\right)$ and let $h_{1}^{\prime}=\sum_{p \in P} n_{p} h_{p}^{\prime}$ 
where $n_{p}$ is the product of all primes in $P$ distinct from $p$. Notice that $h_{1}^{\prime} \notin M^{\prime}+p G^{\prime}$ for all $p \in P$. Next let $q$ be the product of all primes in $P$ that do not divide $m$; naturally, $q$ is understood to be 1 if the product is vacuous. Write $h_{1}^{\prime}=z_{1}^{\prime}+m_{1} b+n c+d$ where $z_{1}^{\prime} \in M^{\prime}$ and $d \in D$. Finally, choose $\alpha$ to be that unique integer such that $1 \leq q n+\alpha t \leq t$ and let

$$
x^{\prime}=w+q h_{1}^{\prime}+\alpha h_{0}^{\prime}=z^{\prime}+q z_{1}^{\prime}+\left(m+q m_{1}\right) b+(q n+\alpha t) c+q d .
$$

We shall show that $x^{\prime} \notin M^{\prime}+p G$, for all primes $p$. First observe that if $x^{\prime} \in M^{\prime}+p G^{\prime}$ then $p \leq t$ since $p$ would have to divide $q n+\alpha t \leq t$, and, consequently, $(* *)$ implies $p \in P$. Thus it remains only to prove that $x^{\prime} \notin M^{\prime}+p G^{\prime}$ for all $p \in P$. But recall that if $p \in P$, then either $p \mid m$ and $p \nmid q$ or else $p \nmid m$ and $p \mid q$. In the first instance, $q h_{1}^{\prime} \notin M^{\prime}+p G^{\prime}$ and $w+\alpha h_{0}^{\prime}=z^{\prime}+m b+\alpha t c \in M^{\prime}+p G^{\prime}$; while in the second, $q h_{1}^{\prime} \in M^{\prime}+p G^{\prime}$ and $w+\alpha h_{0}^{\prime}=z^{\prime}+m b+\alpha t c \notin M^{\prime}+p G^{\prime}$. So, in either case, $x^{\prime}=w+q h_{1}^{\prime}+\alpha h_{0}^{\prime} \notin$ $M^{\prime}+p G^{\prime}$ for $p \in P$. Since, as we have now proved, $x^{\prime} \notin M^{\prime}+p G^{\prime}$ for all primes $p$, we know that $b_{1}=\left(m+q m_{1}\right) b+(q n+\alpha t) c+q d$ generates a direct summand of $\langle b\rangle \oplus C$, and therefore $N^{\prime}=M^{\prime} \oplus\left\langle b_{1}\right\rangle=M^{\prime} \oplus\left\langle x^{\prime}\right\rangle$ is the desired direct summand of $G$.

Having now constructed the summand $N^{\prime}=M^{\prime} \oplus\left\langle x^{\prime}\right\rangle$ with $x^{\prime}+H^{\prime}=$ $w+H^{\prime}=\phi(x+H)$, we once again take $\tau: N \rightarrow N^{\prime}$ to be that unique extension of $\pi$ with $\tau(x)=x^{\prime}$. Clearly then conditions (1), (2) and (3) continue to hold with $\tau, N$ and $N^{\prime}$ replacing $\pi, M$ and $M^{\prime}$, respectively. With regard to (4), we need once again to show that

$$
\operatorname{dim}\left[\frac{H \cap(N+p G)}{H \cap(M+p G)}\right]=\operatorname{dim}\left[\frac{H^{\prime} \cap\left(N^{\prime}+p G^{\prime}\right)}{H^{\prime} \cap\left(M^{\prime}+p G^{\prime}\right)}\right]
$$

for all primes $p$. Noting that these spaces can only have dimension 0 or 1 , it suffices to demonstrate that dimension 1 is attained for exactly the same primes $p$. Referring once again to $(*)$ and its analogue in $G$, we see that it is sufficient to prove, for each prime $p$, that $x \in H+M+p G$ if and only if $x^{\prime} \in H^{\prime}+M^{\prime}+p G^{\prime}$. Since, however, $\phi(x+H)=x^{\prime}+H^{\prime}$ and $\phi: G / H \rightarrow G^{\prime} / H^{\prime}$ is an isomorphism, the equivalence of these conditions is obvious.

Although it is evident Lemma 2.5 suffices to prove Theorem 2.4 in the special case where $G$ and $G$ are countable, some further care must be exercised with the infinite combinatorics in order to handle arbitrary infinite rank free groups. We shall also find it convenient first to prove the theorem with the further hypothesis that $\operatorname{rank}(H)=\operatorname{rank}(G)$, and then explain how the case $\operatorname{rank}(H)<$ $\operatorname{rank}(G)$ can be reduced easily to the former situation.

We continue to assume that the hypotheses of Theorem 2.4 hold, and we make the further assumption that $\operatorname{rank}(H)=\operatorname{rank}(G)=m \geq \aleph_{0}$. By Corollary 2.3, we also have $\operatorname{rank}\left(H^{\prime}\right)=\operatorname{rank}\left(G^{\prime}\right)=m$. Then viewing $m$ as the smallest ordinal of cardinality $m$, we fix direct decompositions

$$
G=\bigoplus_{\lambda<m}\left\langle x_{\lambda}\right\rangle \text { and } G^{\prime}=\bigoplus_{\lambda<m}\left\langle x_{\lambda}^{\prime}\right\rangle .
$$


For any subset $I$ of $m$, we let $G(I)=\bigoplus_{\lambda \in I}\left\langle x_{\lambda}\right\rangle$ and $G^{\prime}(I)=\bigoplus_{\lambda \in I}\left\langle x_{\lambda}^{\prime}\right\rangle$. Using the hypothesis $\operatorname{dim}\left(\frac{H+p G}{p G}\right)=\operatorname{dim}\left(\frac{H^{\prime}+p G^{\prime}}{p G^{\prime}}\right)$, we shall choose, for each prime $p$, a fixed isomorphism

$$
\sigma_{p}: \frac{H}{H \cap p G} \rightarrow \frac{H^{\prime}}{H^{\prime} \cap p G^{\prime}}
$$

Our proof of Theorem 2.4 will rely on the inductive construction of a family $\left\{I_{\lambda}\right\}_{\lambda<m}$ of subsets of $m$ with corresponding isomorphisms $\pi_{\lambda}: G\left(I_{\lambda}\right) \rightarrow G^{\prime}\left(I_{\lambda}\right)$ such that the following seven conditions are satisfied:

(i) $I_{\alpha} \subseteq I_{\beta}$ if $\alpha<\beta<m$.

(ii) $I_{\lambda}=\bigcup_{\alpha<\lambda} I_{\alpha}$ if $\lambda$ is a limit ordinal.

(iii) $\left|I_{\lambda}\right| \leq|\lambda| \aleph_{0}$ for all $\lambda<m$.

(iv) $x_{\lambda} \in G\left(I_{\lambda+1}\right)$ and $x_{\lambda}^{\prime} \in G^{\prime}\left(I_{\lambda+1}\right)$ for all $\lambda<m$.

(v) $\pi_{\lambda}(x)+H^{\prime}=\phi(x+H)$ for all $x \in G\left(I_{\lambda}\right)$.

(vi) If $\alpha<\lambda<m$, then $\pi_{\lambda}$ is an extension of $\pi_{\alpha}$.

(vii) For all primes $p$ and all $\lambda<m$,

$$
\sigma_{p}\left(\frac{H \cap\left(G\left(I_{\lambda}\right)+p G\right)}{H \cap p G}\right)=\frac{H^{\prime} \cap\left(G^{\prime}\left(I_{\lambda}\right)+p G^{\prime}\right)}{H^{\prime} \cap p G} .
$$

Indeed, once the existence of the $I_{\lambda}$ 's and $\pi_{\lambda}$ 's is established, $\pi=\sup _{\lambda<m} \pi_{\lambda}$ will obviously be an isomorphism of $G$ onto $G$ that induces $\phi$.

Turning now to the construction of the $I_{\lambda}$ 's and $\pi_{\lambda}$ 's, we suppose that $\beta<m$ and that for all $\lambda<\beta$ we have $I_{\lambda}$ and $\pi_{\lambda}$ satisfying conditions (i)-(vii). Notice that if we set $I_{0}=\varnothing$, then $G\left(I_{0}\right)=0=G\left(I_{0}\right)$ and that all the conditions (i)(vii) are either vacuous or trivial, so we take $I_{0}=\varnothing$. If $\beta$ is a limit ordinal, we comply with (ii) by taking $I_{\beta}=\bigcup_{\alpha<\beta} I_{\alpha}$ and also satisfy (v) and (vi) by letting $\pi_{\beta}=\sup _{\alpha<\beta} \pi_{\alpha}$. Since (iv) is irrelevant in this case and since (vii) involves an inductive condition (that is, a condition preserved by ascending unions), it is clear that this choice of $I_{\beta}$ and $\pi_{\beta}$ satisfies (i)-(vii). The construction where $\beta=\alpha+1$ is, of course, more difficult and relies heavily on Lemma 2.5. Assume then that $\beta=\alpha+1$, and let $M_{0}=G\left(I_{\alpha}\right)$ and $M_{0}^{\prime}=G^{\prime}\left(I_{\alpha}\right)$. The significance of the condition (vii) is that it implies that

$$
\operatorname{dim}\left[\frac{H}{H \cap\left(M_{0}+p G\right)}\right]=\operatorname{dim}\left[\frac{H^{\prime}}{H^{\prime} \cap\left(M_{0}^{\prime}+p G^{\prime}\right)}\right] .
$$

Notice also that, since $\operatorname{rank}(H)=\operatorname{rank}\left(H^{\prime}\right)=m$, condition (iii) insures us that $\operatorname{rank}\left(M_{0}\right)<\operatorname{rank}(H)$ and $\operatorname{rank}\left(M_{0}^{\prime}\right)<\operatorname{rank}\left(H^{\prime}\right)$. Thus the hypotheses of Lemma 2.5 are satisfied, and therefore there is an isomorphism $\tau_{1}: M_{1} \rightarrow M_{1}^{\prime}$ extending $\tau_{0}=\pi_{\alpha}$ and inducing $\phi$ on $M_{1}$ where $M_{1}$ and $M_{1}^{\prime}$ are direct summands of $G$ and $G$, respectively, such that $x_{\alpha} \in M_{1}, x_{\alpha}^{\prime} \in M_{1}^{\prime}$ and both $M_{1} / M_{0}$ and $M_{1}^{\prime} / M_{0}^{\prime}$ are finitely generated. But, of course, there is no reason to expect either $M_{1}$ or $M_{1}^{\prime}$ to be of the form $G(I)$ or $G^{\prime}(I)$. On the other hand, we can certainly pick a finite subset $\left\{\lambda_{1}, \ldots, \lambda_{n}\right\}$ of $m$ such that 
$N_{1}=M_{0} \oplus\left\langle x_{\lambda_{1}}\right\rangle \oplus \cdots \oplus\left\langle x_{\lambda_{n}}\right\rangle$ and $N_{1}^{\prime}=M_{0}^{\prime} \oplus\left\langle x_{\lambda_{1}}^{\prime}\right\rangle \oplus \cdots \oplus\left\langle x_{\lambda_{n}}^{\prime}\right\rangle$ contain $M_{1}$ and $M_{1}^{\prime}$, respectively. Since the hypotheses of Lemma 2.5 remain intact for $\tau_{1}: M_{1} \rightarrow M_{1}^{\prime}$, we can once again find an appropriate extension $\tau_{2}: M_{2} \rightarrow M_{2}^{\prime}$ where the direct summands $M_{2}$ and $M_{2}^{\prime}$ contain $N_{1}$ and $N_{1}^{\prime}$, respectively, and both $M_{2} / M_{1}$ and $M_{2}^{\prime} / M_{1}^{\prime}$ are finitely generated. Continuing in the manner, we can obviously build up an appropriate ascending sequence $\tau_{n}: M_{n} \rightarrow M_{n}^{\prime}$ of such isomorphisms in such a manner that $M=\bigcup_{n<\omega} M_{n}=G(I)$ and $M^{\prime}=\bigcup_{n<\omega} M_{n}^{\prime}=G^{\prime}(I)$ where $I$ is a subset of $m$ with $I \backslash I_{\alpha}$ countable. Unfortunately, we cannot simply take $I_{\alpha+1}=I$ and $\pi_{\alpha+1}=\sup _{n<\omega} \tau_{n}$ because condition (vii) may fail to be preserved in the above construction of the $\tau_{n}$ 's. In fact, Lemma 2.5 cannot be strengthened directly in such a fashion that (vii) will continue to hold for the extensions $\tau_{n}$. Nevertheless, it is possible to modify the construction of the $\tau_{n}$ 's in such a fashion that (vii) will be recaptured when we take their supremum. Indeed, for any $n<\omega$ and any prime $p$, there will be finite subsets $S_{n, p}$ and $S_{n, p}^{\prime}$ of $H$ and $H^{\prime}$, respectively, such that

$$
\begin{aligned}
\sigma_{p}\left(\frac{H \cap\left(M_{n}+p G\right)}{H \cap p G}\right) & \subseteq \frac{H^{\prime} \cap\left(M_{n-1}^{\prime}+p G^{\prime}\right)}{H^{\prime} \cap p G^{\prime}}+\frac{\left\langle S_{n, p}^{\prime}\right\rangle+\left(H^{\prime} \cap p G^{\prime}\right)}{H^{\prime} \cap p G^{\prime}} \\
& \subseteq \sigma_{p}\left(\frac{H \cap\left(M_{n}+p G\right)}{H \cap p G}+\frac{\left\langle S_{n, p}\right\rangle+(H \cap p G)}{H \cap p G}\right) .
\end{aligned}
$$

Now fix an enumeration $p_{1}, p_{2}, \ldots, p_{n}, \ldots$ of the set of all rational primes. We then need only modify the construction of $\tau_{n+1}: M_{n+1} \rightarrow M_{n+1}^{\prime}$ so that $M_{n+1}$ and $M_{n+1}^{\prime}$ also contain, respectively, the finite sets $\bigcup_{p \leq p_{n}}\left(S_{1, p} \cup \cdots \cup S_{n, p}\right)$ and $\bigcup_{p \leq p_{n}}\left(S_{1, p}^{\prime} \cup \cdots \cup S_{n, p}^{\prime}\right)$. With this reformulation of the construction of the $\tau_{n}$ 's, we can now take $\pi_{\alpha+1}=\sup _{n<\omega} \tau_{n}$ and $I_{\alpha+1}=I$ with all the conditions (i)-(vii) being satisfied for $\lambda \leq \alpha+1$. This completes the inductive construction of the $I_{\lambda}$ 's and $\pi_{\lambda}$ 's, and thus we have proved Theorem 2.4 under the restriction that $\operatorname{rank}(H)=\operatorname{rank}(G)$.

It remains to prove the theorem in the case where $\aleph_{0} \leq \operatorname{rank}(H)<\operatorname{rank}(G)=$ $m$. By Corollary 2.3, we still have $\operatorname{rank}\left(H^{\prime}\right)=\operatorname{rank}(H)<\operatorname{rank}(G)=\operatorname{rank}\left(G^{\prime}\right)$. Adopting the notation of the foregoing argument, we shall first show that there is a subset $I$ of $m$ such that $|I|=\operatorname{rank}(H), H \subseteq G(I), H^{\prime} \subseteq G^{\prime}(I)$ and $\phi(G(I) / H)=G^{\prime}(I) / H^{\prime}$. Indeed, we need only take $I=\bigcup_{n<w} I_{n}$ where $\left\{I_{n}\right\}_{n<w}$ is an ascending sequence of subsets of $m$ such that $H \subseteq G\left(I_{0}\right),\left|I_{n}\right|=\operatorname{rank}(H)$ for all $n, G\left(I_{n+1}\right) / H^{\prime} \supseteq \phi\left(G\left(I_{n}\right) / H\right)$ if $n$ is even and $G\left(I_{n+1}\right) / H \supseteq$ $\phi^{-1}\left(G^{\prime}\left(I_{n}\right) / H^{\prime}\right)$ if $n$ is odd. Since $\operatorname{rank}(H)$ is infinite, it is clear that such a sequence of $I_{n}$ 's does exist. Now let $G_{1}=G(I), G_{1}^{\prime}=G^{\prime}(I)$ and note that we have a direct decomposition $G=G_{1} \oplus G_{2}$. Because

$$
\operatorname{rank}(H)=\operatorname{rank}\left(G_{1}\right)=\operatorname{rank}\left(G_{1}^{\prime}\right)=\operatorname{rank}\left(H^{\prime}\right)
$$


and

$$
\operatorname{dim}\left(\frac{H+p G_{1}}{p G_{1}}\right)=\operatorname{dim}\left(\frac{H+p G}{p G}\right)=\operatorname{dim}\left(\frac{H^{\prime}+p G^{\prime}}{p G}\right)=\operatorname{dim}\left(\frac{H^{\prime}+p G_{1}^{\prime}}{p G_{1}^{\prime}}\right)
$$

and because $\phi$ maps $G_{1} / H$ onto $G_{1}^{\prime} / H^{\prime}$, the special case of Theorem 2.4 already proved yields an isomorphism $\pi_{1}: G_{1} \rightarrow G_{1}^{\prime}$ such that $\pi_{1}(x)+H^{\prime}=$ $\phi(x+H)$ for all $x \in G_{1}$. Clearly

$$
K / H^{\prime}=\phi\left(\frac{G_{2}+H}{H}\right)
$$

is a free subgroup of $G / H^{\prime}$ such that $G^{\prime}=G_{1}^{\prime}+K$ and $G_{1}^{\prime} \cap K=H^{\prime}$. By the projectivity of free groups, $K=H^{\prime} \oplus G_{2}$ and a routine argument shows that $G=G_{1}^{\prime} \oplus G_{2}^{\prime}$. But then there is a well-defined map $\pi_{2}\left(g_{2}\right)=g_{2}^{\prime}$ from $G_{2}$ onto $G_{2}$ defined by the correspondence

$$
g_{2} \rightarrow g_{2}+H \rightarrow \phi\left(g_{2}+H\right)=g_{2}^{\prime}+H^{\prime} \rightarrow g_{2}^{\prime},
$$

and $\pi=\pi_{1} \oplus \pi_{2}$ is the desired isomorphism from $G$ to $G$ inducing $\phi$.

\section{THE STACKED BASES THEOREM}

The following theorem was conjectured by Kaplansky [6] and first proved by J. Cohen and H. Gluck.

Theorem 3.1 [2]. If $H$ is a subgroup of the free group $G$ such that $G / H$ is a direct sum of cyclic groups, then there is a basis $\left\{x_{i}\right\}_{i \in I}$ for $G$ and a corresponding family $\left\{n_{i}\right\}_{i \in I}$ of nonnegative integers such that $\left\{n_{i} x_{i}\right\}_{i \in I}$ is a basis for $H$.

When the conclusion of Theorem 3.1 holds, we say that the basis $\left\{x_{i}\right\}_{i \in I}$ of $G$ is "stacked" over the basis $\left\{n_{i} x_{i}\right\}_{i \in I}$ of $H$. Of course, in the special case where $G / H$ is finite, this is just the classical Proposition 2.1 above. But, as noted in [2], there is no uniqueness in the choice of the $n_{i}$ 's when $G$ has infinite rank. Our plan of attack is to derive Theorem 3.1 from Theorem 1.1 by first establishing an appropriate existence theorem. Indeed we need only show that we can construct a free group $G^{\prime}$ with basis $\left\{x_{i}^{\prime}\right\}$ "stacked" over a basis of a subgroup $H^{\prime}$ where $G / H^{\prime} \cong G / H$ and $\operatorname{dim}\left(\frac{H^{\prime}+p G^{\prime}}{p G^{\prime}}\right)=\operatorname{dim}\left(\frac{H+p G}{p G}\right)$ for all primes $p$. Then Theorem 1.1 guarantees the existence of an isomorphism $\pi: G^{\prime} \rightarrow G$ such that $\pi\left(H^{\prime}\right)=H$, and if $x_{i}=\pi\left(x_{i}^{\prime}\right)$, the basis $\left\{x_{i}\right\}_{i \in I}$ of $G$ will be "stacked" over a basis of $H$. Observe that there is no loss of generality in assuming, as we do, that $A=G / H$ is torsion and that $|A|=|G|$. Hence Theorem 3.1 is a corollary of the following existence theorem.

Theorem 3.2. Suppose $H$ is a subgroup of the free group $G$ such that the infinite group $A=G / H$ is a direct sum of finite cyclic groups and that $|A|=|G|$. For each prime $p$, let $\delta(p)=\operatorname{dim}\left(\frac{H+p G}{p G}\right)$ and let $m=|A|$. Then the cardinal valued function $\delta$ satisfies the following two conditions:

(1) $m=\max \{\delta(p), \operatorname{dim}(A[p])\}$ for all primes $p$. 
(2) $m=\sup _{p \in S} \delta(p)$ for each infinite set of primes $S$.

Conversely, if the infinite group $A$ is a direct sum of finite cyclic groups and if $\delta$ is a cardinal valued function satisfying (1) and (2), then there exists a free group $G=\bigoplus_{i \in I}\left\langle x_{i}\right\rangle$ of rank $m$ and a subgroup $H=\bigoplus_{i \in I}\left\langle n_{i} x_{i}\right\rangle$ such that $G / H \cong A$ and $\operatorname{dim}\left(\frac{H+p G}{p G}\right)=\delta(p)$ for all primes $p$.

Proof. Recall that $A[p]=\{x \in A: p x=0\}$ and that if $A$ is a direct sum of finite cyclic groups, then $\operatorname{dim}(A[p])=\operatorname{dim}(A / p A)$. Furthermore, since $A$ is an infinite torsion $\operatorname{group}, \operatorname{rank}(H)=\operatorname{rank}(G)=|A|$. Thus (1) is an immediate consequence of our earlier formula $\operatorname{rank}(G)=\operatorname{dim}\left(\frac{H+p G}{p G}\right)+\operatorname{dim}(A / p A)$; and, of course, (2) now follows from Proposition 2.2.

Conversely, assume that $\delta$ is a cardinal valued function defined on the set $\mathbf{P}$ of rational primes satisfying (1) and (2) where $|A|=m \geq \aleph_{0}$ and $A$ is a direct sum of finite cyclic groups. Viewing $m$ as an ordinal, we set $G=\bigoplus_{\lambda<m}\left\langle x_{\lambda}\right\rangle$ where $O\left(x_{\lambda}\right)=\infty$ for each $\lambda$. Let $P(m)$ denote the set of all subsets of $m$. We now wish to observe that the existence of the desired positive integers $n_{\lambda}$ will follow once we show that there is a function $\phi: \mathbf{P} \rightarrow P(m)$ satisfying the conditions:

(i) $|\phi(p)|=\operatorname{dim}(A[p])$ for all $p \in \mathbf{P}$.

(ii) $|m \backslash \phi(p)|=\delta(p)$ for all $p \in \mathbf{P}$.

(iii) If $\lambda<m$, then $\{p \in \mathbf{P}: \lambda \in \phi(p)\}$ is finite.

The fundamental idea here is simple, namely, the positive integer $n_{\lambda}$ is to be divisible by the prime $p$ if and only if $\lambda \in \phi(p)$. Thus, (iii) is merely a reformulation of the basic fact that a positive integer $n_{\lambda}$ can be divisible by at most finitely many primes. If we are to take $H=\bigoplus_{\lambda<m}\left\langle n_{\lambda} x_{\lambda}\right\rangle$, then condition (ii) reflects the fact that we desire the following

$$
\operatorname{dim}\left(\frac{H+p G}{p G}\right)=\left|\left\{\lambda<m: p \nmid n_{\lambda}\right\}\right|
$$

to be equal to $\delta(p)$. Finally, condition (i) can be viewed as no more than the assertion that $n_{\lambda}$ contributes a nontrivial cyclic summand to the $p$-primary component of $G / H$ if and only if $p \mid n_{\lambda}$. (By specifying the $p$-height of the individual members of a fixed basis of $A[p]$, we can be completely explicit about what power of $p$ is to divide $n_{\lambda}$ when $\lambda \in \phi(p)$.)

In constructing $\phi: \mathbf{P} \rightarrow P(m)$ satisfying the above conditions, it is helpful to make the following trivial observation: If $\mathbf{P}$ is partitioned into disjoint subsets $S_{1}$ and $S_{2}$ and if we have functions $\phi_{j}: S_{j} \rightarrow P(m) \quad(j=1,2)$ such that each $\phi_{j}$ satisfies the requisite conditions (i), (ii) and (iii) for all $p \in S_{j}$, then the piecewise extension to $\mathbf{P}$ of $\phi_{1}$ and $\phi_{2}$ will yield a function with all the desired properties. In particular, it suffices to define $\phi$ separately on the subsets $S_{1}=\{p \in \mathbf{P}: \delta(p)<m\}$ and $S_{2}=\{p \in \mathbf{P}: \delta(p)=m\}$.

If $S_{1} \neq \varnothing$, then we have a sequence (possibly finite) of cardinals $m_{0}$, $m_{1}, \ldots, m_{k}, \ldots$ strictly smaller than $m$ and a corresponding partition $P_{0}$, $P_{1}, \ldots, P_{k}, \ldots$ of $S_{1}$ where $P_{k}=\left\{p \in \mathbf{P}: \delta(p)=m_{k}\right\}$ is not empty. By 
condition (2), each $P_{k}$ is finite. Following the customary convention, we view each $m_{k}$ as the smallest initial segment of $m$ having cardinality $m_{k}$. We now define $\phi$ on $S_{1}$ by setting $\phi(p)=\left\{\lambda<m: \lambda \geq m_{k}\right\}$ when $p \in P_{k}$. Clearly (i) and (ii) are satisfied for all $p \in S_{1}$ since (1) implies $|\phi(p)|=m$ whenever $\delta(p)<m$ and, of course, $|m \backslash \phi(p)|=\left|\left\{\lambda<m: \lambda<m_{k}\right\}\right|=m_{k}=\delta(p)$ if $p \in P_{k}$. With regard to (iii), certainly $\left\{p \in S_{1}: \lambda \in \phi(p)\right\}$ is finite if $S_{1}$ is finite; while if $S_{1}$ is infinite, then $\left\{p \in S_{1}: \lambda \in \phi(p)\right\}=\bigcup\left\{P_{k}: \lambda \geq m_{k}\right\}$ remains finite since the supremum of infinitely many $m_{k}$ 's must equal $m$. The definition of $\phi$ on $S_{2}$ is even more straightforward. Since $m$ is infinite and $S_{2}$ is at most countable, we can partition $m$ into pairwise disjoint subsets $I_{p}$, $p \in S_{2} \cup\{0\}$, where $\left|I_{0}\right|=m$ and $\left|I_{p}\right|=\operatorname{dim}(A[p])$ for $p \in S_{2}$. We then define $\phi(p)=I_{p}$ for all $p \in S_{2}$. Condition (i) is immediate for $p \in S_{2}$ by the choice of $I_{p}$ and (ii) holds since $|m \backslash \phi(p)|=\left|m \backslash I_{p}\right|=m=\delta(p)$ for $p \in S_{2}$. Also, (iii) is trivial since $\left\{p \in S_{2}: \lambda \in \phi(p)\right\}$ consists at most of a single prime. Thus, $\phi$ is satisfactorily defined and the proof of Theorem 3.2 is complete.

Remark 3.3. If we first avail ourselves of some routine infinite combinatorics, the proof of the stacked bases theorem can be derived from the special case of Theorem 1.1 where $\operatorname{rank}(G)=\aleph_{0}$.

\section{THE GENERAL EXISTENCE THEOREM}

In this section, $A$ will denote an arbitrary infinite abelian group with maximal torsion subgroup $A_{t}$. Once again we consider free resolutions

$$
0 \rightarrow H \rightarrow G \stackrel{\theta}{\rightarrow} A \rightarrow 0
$$

where $H=\operatorname{Ker} \theta$. We wish to describe precisely the permissible values of the function $\delta(p)=\operatorname{dim}\left(\frac{H+p G}{p G}\right)$ associated with any such resolution of $A$ and thereby classify the free resolutions of any abelian group $A$ by means of the numerical invariants $\delta(p)$. Towards this end, we shall associate with each abelian group $A$ a cardinal number $m_{A}$ intimately related to the free resolutions of $A$. We first choose a subgroup $B$ of minimal cardinality with $A / B$ free. If $B$ is infinite, then we let $m_{A}=|B|$. If $B$ is finite, then we decompose it into a direct sum $B=\left\langle b_{1}\right\rangle \oplus \cdots \oplus\left\langle b_{m}\right\rangle$ where the $b_{i}$ 's are nonzero, $O\left(b_{i}\right) \mid O\left(b_{i+1}\right)$ for $i=1, \ldots, m-1$ and we take $m_{A}=m$. In either case, $A_{t}=B_{t}$ and $m_{A}=m_{B}$. Notice that $A$ is free if and only if $m_{A}=0$.

Proposition 4.1. If (1) is a free resolution of $A$, then there exist direct decompositions $G=G_{1} \oplus H_{2}$ and $H=H_{1} \oplus H_{2}$ where $H_{1} \subseteq G_{1}$ and $\operatorname{rank}\left(H_{1}\right)=m_{A}$. Furthermore, for each prime $p$,

$$
\operatorname{dim}\left(\frac{H+p G}{p G}\right)=\operatorname{dim}\left(\frac{H_{1}+p G_{1}}{p G_{1}}\right)+\operatorname{rank}\left(H_{2}\right) .
$$

Proof. Write $A=B \oplus F$ where $F$ is free and $B$ is as in our definition of $m_{A}$ above. Since $G / H \cong B \oplus F$, we have a direct decomposition $G=G_{0} \oplus F^{\prime}$ where $H \subseteq G_{0}, F^{\prime} \cong F$ and $G_{0} / H \cong B$. If $B$ is finite, then a routine argument yields 
a decomposition $G_{0}=G_{1}^{\prime} \oplus H_{2}$ where $G_{1}^{\prime}$ has finite rank and $H_{2} \subseteq H$. But then an application of Proposition 2.1 shows that we may assume that both $G_{1}^{\prime}$ and $H_{1}=G_{1}^{\prime} \cap H$ have $\operatorname{rank} m_{A}$. Thus, in this case, we need only take $G_{1}=G_{1}^{\prime} \oplus F^{\prime}$ in order to obtain the desired decompositions. Suppose that $B$ is infinite and hence, since $G_{0} / H \cong B$, that $\operatorname{rank}\left(G_{0}\right) \geq|B|$. If we were to have $\operatorname{rank}(H)<|B|$, then there would exist a direct decomposition $B=B_{1} \oplus F_{1}$ with $F_{1}$ free and $\left|B_{1}\right|<|B|$. This, however, would contradict the choice of $B$ and therefore we conclude that $\operatorname{rank}(H) \geq|B|$. If $\operatorname{rank}(H)=|B|=m_{A}$, then we may take $H_{2}=0$. Consequently, we may further assume that $\operatorname{rank}(H)>|B|$. Then, by Corollary 1.3, we have a direct decomposition $G_{0}=G_{1}^{\prime \prime} \oplus H_{2}$ with $\operatorname{rank}\left(G_{1}^{\prime \prime}\right)=|B|, H_{2} \subseteq H$ and $\operatorname{rank}\left(H_{2}\right)>|B|$. Thus if $G_{1}=G_{1}^{\prime \prime} \oplus F^{\prime}$ and $H_{1}=H \cap G_{1}^{\prime \prime}$, then we once again have the desired direct decompositions. The final assertion of the proposition follows from the obvious isomorphism $\left(\frac{H+p G}{p G}\right) \cong\left(\frac{H_{1}+p G_{1}}{p G_{1}}\right) \oplus\left(H_{2} / p H_{2}\right)$ and the fact that $\operatorname{rank}\left(H_{2}\right)=\operatorname{dim}\left(H_{2} / p H_{2}\right)$.

Because of the reduction effected by Proposition 4.1, we need only characterize the permissible values of $\delta(p)=\operatorname{dim}\left(\frac{H+p G}{p G}\right)$ for free resolutions (1) subject to the further restriction that $\operatorname{rank}(H)=m_{A}$. The remainder of this section will be devoted to the proof of the following theorem.

Theorem 4.2. Suppose (1) is a free resolution of $A$ with $\operatorname{rank}(H)=m_{A}$ and, for each prime $p$, let $\delta(p)=\operatorname{dim}\left(\frac{H+p G}{p G}\right)$. Then the following two conditions hold:

(i) For all primes $p, m_{A}=\delta(p)+\operatorname{dim}\left(A_{t} / p A_{t}\right)$.

(ii) For any infinite set of primes $S, \sup _{p \in S} \delta(p)=m_{A}$.

Conversely, if $\delta$ is a cardinal valued function on $\mathbf{P}$ that satisfies (i) and (ii), then there exists a free resolution (1) of $A$ with $\operatorname{rank}(H)=m_{A}$ such that $\operatorname{dim}\left(\frac{H+p G}{p G}\right)=\delta(p)$ for all primes $p$.

We have already seen, in Proposition 2.2, that condition (ii) is a necessary one. Given a free resolution (1) with $\operatorname{rank}(H)=m_{A}$, we take $G$ to be the complete inverse image under $\theta$ of the torsion subgroup $A_{t}$. Then we will have a commutative diagram

$$
\begin{aligned}
& 0 \rightarrow H \rightarrow G^{\prime} \rightarrow A_{t} \rightarrow 0 \\
& \| \begin{array}{l}
\downarrow \\
0
\end{array} \quad+H \rightarrow G \rightarrow A \rightarrow 0
\end{aligned}
$$

where $G / G^{\prime} \cong A / A_{t}$. Since $G / G^{\prime}$ is torsion free, $p G \cap H=p G \cap H$ and consequently

$$
\frac{H+p G^{\prime}}{p G} \cong \frac{H}{H \cap p G^{\prime}}=\frac{H}{H \cap p G} \cong \frac{H+p G}{p G} .
$$

But then formula (3) from $\S 1$ yields, for each prime $p, \operatorname{rank}\left(G^{\prime}\right)=\delta(p)+$ $\operatorname{dim}\left(A_{t} / p A_{t}\right)$. Since $G^{\prime} / H$ being torsion implies $\operatorname{rank}\left(G^{\prime}\right)=m_{A}$, it follows that (i) is also a necessary condition for the existence of such a free resolution of $A$. 
Now assume that $\delta$ is any function satisfying (i) and (ii) of Theorem 4.2 and once again write $A=B \oplus F$ where $F$ is free and $B$ is of minimal cardinality. Clearly it suffices to construct a free resolution $0 \rightarrow H \rightarrow G \rightarrow B \rightarrow 0$ where $\operatorname{dim}\left(\frac{H+p G^{\prime}}{p G^{\prime}}\right)=\delta(p)$ for all $p$, for then we can obtain the desired resolution of $A$ with $G=G \oplus F$. If $m_{A}$ is finite, then $\delta$ is uniquely determined by condition (i) and hence it is enough, in this case, to exhibit a free resolution of $B$ with kernel of rank $m_{A}$. But this is trivial since we can take $\boldsymbol{G}^{\prime}=\left\langle x_{1}\right\rangle \oplus \cdots \oplus\left\langle x_{m}\right\rangle$ and $H=\left\langle n_{1} x_{1}\right\rangle \oplus \cdots \oplus\left\langle n_{m} x_{m}\right\rangle$ with $n_{i}=O\left(b_{i}\right)$, where the $b_{i}$ 's are as in our earlier representation of $B$. Thus we may assume that $m_{A}$ is infinite and, replacing $A$ by $B$, that $m_{A}=|A|$.

In all subsequence discussion in this section, $m$ denotes the cardinality of the infinite group $A$ and $m_{A}=m$. We shall require some preliminary observations before we can complete the proof of Theorem 4.2. Let us consider a well-ordered set $\left\{x_{\alpha}\right\}_{\alpha<m}$ of generators of $A$ and take $G$ to be the abelian group freely generated by the corresponding set of elements $\left\{g_{\alpha}\right\}_{\alpha<m}$. Then let $H=\operatorname{Ker} \theta$ where $\theta: G \rightarrow A$ is the unique homomorphism determined by the requirement that $\theta\left(g_{\alpha}\right)=x_{\alpha}$ for all $\alpha<m$. For each $\alpha$, we take $C_{\alpha}$ to be the subgroup of $A$ generated by all $x_{\beta}$ with $\beta<\alpha$ and set $G_{\alpha}=\bigoplus_{\beta<\alpha}\left\langle g_{\beta}\right\rangle$. The following elementary result is crucial to our proof of Theorem 4.2 .

Lemma 4.3. For each prime $p$ and each $a<m$, let

$$
V_{\alpha}^{(p)}=\left(\frac{H+p G}{p G}\right) \cap\left(\frac{G_{\alpha}+p G}{p G}\right) .
$$

Then $V_{\alpha+1}^{(p)}=V_{\alpha}^{(p)}$ if and only if $x_{\alpha} \notin C_{\alpha}+p A$.

Proof. If $V_{\alpha+1}^{(p)} \neq V_{\alpha}^{(p)}$, then there must exist an integer $t$, not divisible by $p$, such that $t g_{\alpha} \in G_{\alpha}+p G+H$. Clearly we may take $t=1$ and therefore it follows, by applying $\theta$, that $x_{\alpha} \in C_{\alpha}+p A$. Conversely, if $x_{\alpha} \in C_{\alpha}+p A$, then $g_{\alpha} \in G_{\alpha}+p G+H$ and hence $V_{\alpha+1}^{(p)} \neq V_{\alpha}^{(p)}$.

In order to exploit Lemma 4.3 in the construction of free resolutions of $A$, we shall also require the following technical lemma.

Lemma 4.4. Suppose $C$ is a subgroup of $A$ generated by fewer than $m$ elements and let $S$ be a finite set of primes such that $\operatorname{dim}\left(A_{t} / p A_{t}\right)=m$ for all $p \in S$. Then to each $a \in A$ there corresponds an $x \in A$ such that $a \in\langle C, x\rangle, x \notin$ $C+p A$ for all $p \in S$ and $x \in C+p A$ whenever $p$ is a prime with $p \notin S$ but $a \in p A$.

Proof. Let $S=\left\{p_{1}, \ldots, p_{k}\right\}$. Proceeding by induction, let us suppose that there is an element $y \in A$ such that $y \notin C+p_{i} A$ for $i \leq k-1$ and $a-t y \in C$ where $t$ is a positive integer each prime factor of which occurs among the $p_{i}$ 's with $i \leq k-1$. We shall show that there is an $x \notin C+p_{k} A$ such that $y-p_{k}^{n} x \in C$ for some nonnegative integer $n$. This is obvious if there is a nonnegative $n$ such that $y \in C+p_{k}^{n} A$ and $y \notin C+p_{k}^{n+1} A$. Thus we may 
assume that $y \in C+p_{k}^{l} A$ for all $l<w$. Since $C$ is generated by fewer than $m$ elements and $\operatorname{dim}\left(A_{t} / p_{k} A_{t}\right)=m$, it is clear that there is a $w \notin C+p_{k} A$ having finite order $p_{k}^{n}$. Now write $y=c+p_{k}^{n} z$ where $c \in C$. If $z \notin C+p_{k} A$, then we may take $x=z$; while if $z \in C+p_{k} A$, then $x=z+w$ will have the desired property. Since $a-p_{k}^{n} t x \in C$, it is easily checked that $x$ has all the desired properties.

We are now in position to complete the proof of Theorem 4.2. Let us assume then that we have a function $\delta$ satisfying (i) and (ii) of that theorem. Let $S=\{p \in \mathbf{P}: \delta(p)<m\}$ and observe that (i) implies that $S$ is contained in $T(A)=\left\{p \in \mathbf{P}: \operatorname{dim}\left(A_{t} / p A_{t}\right)=m\right\}$. Thus if $T(A)=\varnothing$, then any free resolution (1) of $A$ with $\operatorname{rank}(H)=m$ will serve our purpose. Surprisingly the special case where $T(A)$ consists of but a single prime requires ad hoc treatment, and so we shall first establish the existence of the desired resolution (1) corresponding to $\delta$ under the hypothesis that $T(A)$ contains at least two distinct primes. We begin by fixing a well-ordering $\left\{a_{\alpha}\right\}_{\alpha<m}$ of the elements of $A$. Our proof of the existence of the desired free resolution of $A$ will be based on the simultaneous inductive construction of a family $B_{\alpha}$ of subsets of $A$ and a family of functions $\delta^{(\alpha)}: \mathbf{P} \rightarrow m$ satisfying the following conditions:

(a) $B_{0}=\varnothing$ and $\delta^{(0)}(p)=0$ for all primes $p$.

(b) The elements $x_{\beta}$ of $B_{\alpha}$ are indexed by the ordinal $\alpha$ and $\delta^{(\alpha)}(p) \leq \alpha$ for all primes $p$.

(c) If $\alpha$ is a limit ordinal, then $B_{\alpha}=\bigcup_{\beta<\alpha} B_{\beta}$ and $\delta^{(\alpha)}(p)=\sup _{\beta<\alpha} \delta^{(\beta)}(p)$ for each prime $p$.

(d) $C_{\alpha}=\left\langle B_{\alpha}\right\rangle$ contains all $\alpha_{\beta}$ 's with $\beta<\alpha$.

Of course, some further conditions must be specified governing the transition from $B_{\alpha}$ to $B_{\alpha+1}$. Towards this end, suppose that $B_{\alpha}$ and $\delta^{(\alpha)}$ have been constructed. We associate with $S=\{p \in \mathbf{P}: \delta(p)<m\}$ a sequence (possibly finite or even empty) of cardinals $m_{0}, m_{1}, \ldots, m_{k}, \ldots$ strictly smaller than $m$ and a corresponding partition $P_{0}, P_{1}, \ldots, P_{k}, \ldots$ of $S$ where $P_{k}=\{p \in$ P: $\left.\delta(p)=m_{k}\right\}$ is not empty. The $P_{k}$ 's are finite by (ii), and with each $\alpha<m$ we let $S_{\alpha}=\bigcup\left\{P_{k}: m_{k} \leq \alpha\right\}$. Notice that each $S_{\alpha}$ is also finite since when $S$ is infinite, (ii) ensures that the supremum of any infinite number of the $m_{k}$ 's will equal $m$. Next let $T_{\alpha}=\left\{p \in S_{\alpha}: \delta^{(\alpha)}(p)=\delta(p)\right\}$. We now rely on Lemma 4.4 to describe the construction of $B_{\alpha+1}$ and $\delta^{(\alpha+1)}$ by imposing the following further condition:

(e) Let $B_{\alpha+1}=B_{\alpha} \cup\left\{x_{\alpha}\right\}$ where $x_{\alpha}$ is chosen so that $a_{\alpha} \in\left\langle C_{\alpha}, x_{\alpha}\right\rangle, x_{\alpha} \notin$ $C_{\alpha}+p A$ for all $p \in T_{\alpha}$ and $x_{\alpha} \in C_{\alpha}+p A$ whenever $p \notin T_{\alpha}$ and $a_{\alpha} \in p A$.

Then define $\delta^{(\alpha+1)}(p)$ for all primes $p$ by

$$
\delta^{(\alpha+1)}(p)= \begin{cases}\delta^{(\alpha)}(p) & \text { if } x_{\alpha} \in C_{\alpha}+p A \\ \delta^{(\alpha)}(p)+1 & \text { if } x_{\alpha} \notin C_{\alpha}+p A\end{cases}
$$


There are now three crucial things to observe about the functions $\delta^{(\alpha)}$. First, since in Lemma $4.3 \operatorname{dim} V_{\alpha}^{(p)}=\bigcup_{\beta<\alpha} V_{\beta}^{(p)}$ when $\alpha$ is a limit and $\operatorname{dim}\left(V_{\alpha+1}^{(p)} / V_{\alpha}^{(p)}\right) \leq 1$, conditions (a)-(e) insure that each $V_{\alpha}^{(p)}$ has a basis indexed by the ordinal $\delta^{(\alpha)}(p)$, provided we take the $x_{\alpha}$ 's as our set of generators of $A$. Since furthermore, $\frac{H+p G}{p G}=\bigcup_{\alpha<m} V_{\alpha}^{(p)}$, this means that $\operatorname{dim}\left(\frac{H+p G}{p G}\right)$ must equal $\left|\sup _{\alpha<m} \delta^{(\alpha)}(p)\right|$ for each prime $p$. Secondly, since $\delta^{(\alpha)}(p) \leq \alpha<$ $\delta(p)$ if $p \in S \backslash S_{\alpha}$, conditions (a)-(e) imply that $\delta^{(\alpha)}(p) \leq \delta(p)$ for all $p \in S$. Thirdly, and this is where the assumption that $|T(A)| \geq 2$ is crucial, if $p$ is a prime such that $\delta^{(\alpha)}(p)<\delta(p)$ for all $\alpha<m$, then $\sup _{\alpha<m} \delta^{(\alpha)}(p)=m$. The point here is that there will be a prime $q \in T(A)$ distinct from $p$ and consequently there must be $m$ choices of $\alpha$ such that $a_{\alpha}$ has order a positive power of $q$. For any such $\alpha, a_{\alpha} \in p A$ and, since $p \notin T_{\alpha}$, condition (e) insures that $V_{\alpha+1}^{(p)} \neq V_{\alpha}^{(p)}$. But if $V_{\alpha+1}^{(p)} \neq V_{\alpha}^{(p)}$ for $m$ different choices of $\alpha$, then $\operatorname{dim}\left(\frac{H+p G}{p G}\right)=\sup _{\alpha<m}\left(\operatorname{dim} V_{\alpha}^{(p)}\right)=m$. Combining these three observations, we conclude that if $p \in S$, then there must be a $\beta$ such that $\delta^{(\alpha)}(p)=\delta(p)$ for all $\alpha \geq \beta$; while if $p \notin S$, then $\sup _{\alpha<m} \delta^{(\alpha)}(p)=m$. Thus if the free resolution (1) arises from the $x_{\alpha}$ 's (as in the discussion preceding Lemma 4.3), then we have the desired property that $\operatorname{dim}\left(\frac{H+p G}{p G}\right)=\delta(p)$ for all primes $p$.

Finally, we must deal with the special situation where $T(A)=\{p\}$. In this case, it suffices to find a resolution (1) with $\operatorname{rank}(H)=m$ and with $\operatorname{dim}\left(\frac{H+p G}{p G}\right)=\delta(p)$ for any given value $0 \leq \delta(p) \leq m$. But $\delta(p)=0$ can be handled by a simplified version of the foregoing argument by selecting the $x_{\alpha}$ 's so that $x_{a} \notin C_{\alpha}+p A$ for all $\alpha$. Then any other value of $\delta(p)$ can be attained by adjoining a superfluous free direct summand of that rank. In greater detail, first choose a free resolution $0 \rightarrow H_{1} \rightarrow G_{1} \rightarrow A \rightarrow 0$ where $\operatorname{rank}\left(H_{1}\right)=\operatorname{rank}\left(G_{1}\right)=m$ and $\operatorname{dim}\left(\frac{H_{1}+p G_{1}}{p G_{1}}\right)=0 ;$ and take $G=G_{1} \oplus G_{2}$ and $H=H_{1} \oplus G_{2}$ where $\operatorname{rank}\left(G_{2}\right)=\delta(p)$.

\section{FURTHER APPLICATIONS}

In this final section, we consider the extent to which direct decompositions of the abelian group $A$ can be lifted to decompositions, or splittings, of a given free resolution of $A$. To fix notation and terminology, let $A=\bigoplus_{i \in I} A_{i}$ and consider a free resolution

$$
0 \rightarrow H \rightarrow G \stackrel{\theta}{\rightarrow} A \rightarrow 0 .
$$

We say that (1) splits over the $A_{i}$ 's if there exists a direct decomposition $G=$ $\bigoplus_{i \in I} G_{i}$ such that, for each $i, A_{i}=\theta\left(G_{i}\right)$. Observe that this implies that $H=\bigoplus_{i \in I}\left(H \cap G_{i}\right)$. Erdös [3] proved that if $A=\bigoplus_{i \in I} A_{i}$ is torsion free, then each free resolution (1) of $A$ splits over the $A_{i}$ 's. This, of course, also follows from Corollary 1.4. On the other hand, when torsion is involved, the situation is radically different. Indeed the free resolution,

$$
0 \rightarrow p q Z \rightarrow Z \rightarrow Z / p q Z \rightarrow 0 \text {, }
$$


where $p$ and $q$ are distinct primes, cannot split over the direct decompositon $Z / p q Z=Z / p Z \oplus Z / q Z$. It might be objected that the failure of splitting in (2) depends on the special fact that $G=Z$ has finite rank. But it is easy to construct examples where splitting fails and the free group $G$ has infinite rank. For example, let $A=A_{1} \oplus A_{2}$ where $A_{1}$ is a direct sum of countably many cyclic groups of fixed prime order $p$ and $A_{2}=Z\left(p^{\infty}\right)$. Then, by Theorem 4.2, $A$ has a free resolution (1) where $\operatorname{rank}(G)=\aleph_{0}$ and $\operatorname{dim}\left(\frac{H+p G}{p G}\right)=0$. Such a free resolution cannot split over $A_{1}$ and $A_{2}$ because, for any free resolution $0 \rightarrow H_{2} \rightarrow G_{2} \rightarrow A_{2} \rightarrow 0$ of $A_{2}=Z\left(p^{\infty}\right), \operatorname{dim}\left(\frac{H_{2}+p G_{2}}{p G_{2}}\right)$ will be infinite.

There is a common thread involved in the two examples considered above. To see this, associate with each abelian group $A$ the set of primes $T(A)=$ $\left\{p \in \mathbf{P}: \operatorname{dim}\left(A_{t} / p A_{t}\right)=m_{A}\right\}$. Whenever $p \in T(A)$, Theorem 4.2 yields a free resolution (1) with $\operatorname{dim}\left(\frac{H+p G}{p G}\right)=0$. But if $p \notin T\left(A_{i}\right)$ for some $i$, then no such resolution can exist for $A_{i}$. Thus, given a direct decomposition $A=\bigoplus_{i \in I} A_{i}$, a necessary condition for every free resolution of $A$ to split over the $A_{i}$ 's is that $T(A) \subseteq T\left(A_{i}\right)$ for all $i \in I$. That this condition is also sufficient is the content of the next theorem.

Theorem 5.1. Let $A=\bigoplus_{i \in I} A_{i}$. Then every free resolution (1) of $A$ splits over the $A_{i}$ 's if and only if $T(A) \subseteq T\left(A_{i}\right)$ for all $i$.

Proof. We begin our proof by demonstrating that $m_{A}=\sum_{i \in I} m_{A_{i}}$, which requires the hypothesis $T(A) \subseteq T\left(A_{i}\right)$ only in case $m_{A}$ is finite. For each $i \in I$, let $A_{i}=B_{i} \oplus F_{i}$ when $F_{i}$ is free and either $B_{i}$ is finite or else $m_{A_{i}}=\left|B_{i}\right|$ is infinite. First assume that $m_{A}$ is finite and note, in this case, that $m_{A}=$ $\max _{p \in \mathrm{P}} \operatorname{dim} A[p]$. In fact, $m_{A}=\operatorname{dim} A[q]$ where $q$ is any prime that divides each $O\left(b_{i}\right)$ in our definition of $m_{A}$. But then the condition $T(A) \subseteq T\left(A_{i}\right)$ insures that $m_{A_{i}}=\operatorname{dim} A_{i}[q]$ for each $i$. Therefore $m_{A}=\sum_{i \in I} m_{A_{i}}$ follows from the fact that $A[q]=\bigoplus_{i \in I} A_{i}[q]$. Now suppose that $m_{A}=|B|$ is infinite where $A / B$ is free. Since $B_{i} / B \cap B_{i} \cong\left(B_{i}+B\right) / B$ is free, we may assume without loss of generality that $B_{i}=B \cap B_{i}$. Thus $B^{\prime}=\bigoplus_{i \in I} B_{i}$ is contained in $B$ and $m_{A}=\left|B^{\prime}\right|$ since clearly $A / B^{\prime}$ is also free. Finally, since either some $B_{i}$ is infinite or else all the $B_{i}$ 's are finite with infinitely many nonzero, it is easily verified that $\left|B^{\prime}\right|=\sum_{i \in I} m_{A_{i}}$.

An immediate application of Proposition 4.1 allows us to restrict ourselves to free resolutions (1) with $\operatorname{rank}(H)=m_{A}$. Since free summands of $A$ are irrelevant to the problem at hand, we may further assume that $m_{A_{i}} \neq 0$ for all $i \in I$. Consequently, $|I| \leq m_{A}$ follows from the observation that $m_{A}=$ $\sum_{i \in I} m_{A_{i}}$. In outline, the proof of the theorem is quite simple. Let $\delta(p)=$ $\operatorname{dim}(H+p G / p G)$ for each prime $p$. We shall show that, for each $i$, there is a free resolution $0 \rightarrow H_{i} \rightarrow G_{i} \rightarrow A_{i} \rightarrow 0$ where the cardinal numbers $\delta^{(i)}(p)=\operatorname{dim}\left(H_{i}+p G_{i} / p G_{i}\right)$ have the property that $\sum_{i \in I} \delta^{(i)}(p)=\delta(p)$ for all primes $p$. Then an application of Theorem 1.1 will show that the resolution (1) is equivalent to the free resolution $0 \rightarrow \bigoplus_{i \in I} H_{i} \rightarrow \bigoplus_{i \in I} G_{i} \rightarrow A \rightarrow 0$ 
and hence the theorem will follow. We shall, however, need to consider three separate cases depending on whether the set $T=\left\{p \in \mathbf{P}: \delta(p)<m_{A}\right\}$ is finite or infinite, and on whether $|I|<m_{A}$ or $|I|=m_{A}$. In each case, we shall define the cardinal valued function $\delta^{(i)}$ directly without reference to any free resolution of $A_{i}$. The desired resolutions of the $A_{i}$ 's will then be obtained by invoking Theorem 4.2. In all three cases, we shall take $\delta^{(i)}(p)=m_{A_{i}}$ whenever $p \notin T$. Thus for $p \notin T, \delta(p)=\sum_{i \in I} \delta^{(i)}(p)$ will be a consequence of the fact that $m_{A}=\sum_{i \in I} m_{A_{i}}$; recall, by Theorem 4.2(i), that $\delta(p)=m_{A}$ if $p \notin T$.

Case 1. $T=\left\{p \in \mathbf{P}: \delta(p)<m_{A}\right\}$ if finite. For $p \in T$, we have $\delta(p)<m_{A}=$ $\sum_{i \in I} m_{A_{i}}$ and hence it is clear that there must exist cardinals $\delta^{(i)}(p)$ such that

$$
\begin{gathered}
\delta^{(i)}(p) \leq m_{A_{i}}, \\
\sum_{i \in I} \delta^{(i)}(p)=\delta(p) .
\end{gathered}
$$

In this case, we require no further restriction on the $\delta^{(i)}(p)$ 's beyond the assumption that (a) and (b) are satisfied for primes $p \in T$ and, of course, $\delta^{(i)}(p)=m_{A_{i}}$, if $p \notin T$.

Case 2. $T$ is infinite and $|I|<m_{A}$. In this case, when $p \in T$ we shall require the $\delta^{(i)}(p)$ 's to satisfy (a) and (b) above and also the further condition:

(c) $\delta^{(i)}(p) \geq \min \left\{m_{A_{i}}, m_{p}\right\}$ where $m_{p}$ is the largest cardinal such that $|I| m_{p} \leq \delta(p)$.

Since $\left|I^{\prime}\right| m_{p} \leq \delta(p)$ where $I^{\prime}=\left\{i \in I: m_{A_{i}}>m_{p}\right\}$ and $\delta(p)<\sum_{i \in I} m_{A_{i}}$, it is clear that it is possible to find cardinals $\delta^{(i)}(p)$ satisfying (a), (b) and (c).

Case 3. $T$ is infinite and $|I|=m_{A}$. By Theorem 4.2 , there is a sequence $\left\{m_{k}\right\}_{k<w}$ of distinct cardinals such that $m_{A}=\sup _{k<w} m_{k}$ and $T=\bigcup_{k<w} P_{k}$ where each $P_{k}=\left\{p \in \mathbf{P}: \delta(p)=m_{k}\right\}$ is nonempty and finite. But since $|I|=m_{A}$, we can also express $I$ as the union of a family $\left\{I_{k}\right\}_{k<w}$ of subsets such that, for each $k,\left|I_{k}\right|=m_{k}$ and $I_{k} \subseteq I_{j}$ if and only if $m_{k} \leq m_{j}$. As in the two previous cases, we take $\delta^{(i)}(p)=m_{A_{i}}$ whenever $p \notin T$. If, however, $p \in T$, we shall require the $\delta^{(i)}(p)$ 's to satisfy (a) and (b) above, as well as the following conditions:

$\left(c^{\prime}\right)$ If $m_{k}^{2} \leq \delta(p)$ and $i \in I_{k}$, then $\delta^{(i)}(p) \geq \min \left\{m_{A_{i}}, m_{k}\right\}$.

Observe that if, for a given $p \in T$, the hypothesis of $\left(\mathrm{c}^{\prime}\right)$ is not vacuous, then there will be a largest $m_{k}$ such that $m_{k}^{2} \leq \delta(p)$ and consequently $I_{j} \subseteq I_{k}$ whenever $m_{j}^{2} \leq \delta(p)$. With this choice of $m_{k}$, we have $\left|I^{\prime}\right| m_{k} \leq \delta(p)$ where $I^{\prime}=\left\{i \in I_{k}: m_{A_{i}}>m_{k}\right\}$. Then since $\delta(p)<\sum_{i \in I} m_{A_{i}}$, it is evident that there do indeed exist cardinals $\delta^{(i)}(p)$ satisfying $(a),(b)$ and $\left(c^{\prime}\right)$. 
The proof of Theorem 5.1 will now be completed by arguing that, regardless which of the three cases holds, we can exhibit, for each $i \in I$, a free resolution $0 \rightarrow H_{i} \rightarrow G_{i} \rightarrow A_{i} \rightarrow 0$ such that $\operatorname{dim}\left(H_{i}+p G_{i} / p G_{i}\right)=\delta^{(i)}(p)$ for all primes $p$. We shall, of course, establish the existence of the desired free resolutions by showing that the conditions of Theorem 4.2 are satisfied for each $A_{i}$. Observe that we must have $T=\left\{p \in \mathbf{P}: \delta(p)<m_{A}\right\}$ contained in $T(A)=\{p \in$ P: $\left.\operatorname{dim}\left(A_{t} / p A_{t}\right)=m_{A}\right\}$ because of (i) of Theorem 4.2. But by the hypothesis $T(A) \subseteq T\left(A_{i}\right)$ and the choice of $\delta^{(i)}(p)=m_{A_{i}}$ whenever $p \notin T$, it certainly follows that $m_{A_{i}}=\delta^{(i)}(p)+\operatorname{dim}\left(\left(A_{i}\right)_{t} / p\left(A_{i}\right)_{t}\right)$ for all primes $p$ and all $i \in I$.

It remains now to show, for each $i$, that $\sup _{p \in S} \delta^{(i)}(p)=m_{A_{i}}$ whenever $S$ is an infinite set of primes. Recalling that $\delta^{(i)}(p)=m_{A_{i}}$ for $p \notin T$, we see that the desired conclusion is trivially satisfied whenever $S$ intersects the complement of $T$ in $\mathbf{P}$. Since that certainly happens if $T$ is finite, we may assume henceforth that $T$ is infinite and that $S \subseteq T$. Observe then that Theorem 4.2 implies that $m_{A}$ is infinite and that $m_{A}=\sup _{p \in S} \delta(p)=$ $\sup _{p \in S} m_{p}$ in Case 2. But then, by condition (c), either $\delta^{(i)}(p)=m_{A_{i}}$ for some $p \in S$ or else $\sup _{p \in S} \delta^{(i)}(p)=m_{A}=m_{A_{i}}$. In Case $3, \sup _{k<\omega} m_{k}=m_{A}=$ $\sup _{p \in S} \delta(p)$, and, by definition of the $I_{k}$ 's, each $i \in I$ is contained in infinitely many of the $I_{k}$ 's. Thus condition ( $\left.\mathrm{c}^{\prime}\right)$ implies, for any fixed $i \in I$, that either $\delta^{(i)}(p)=m_{A_{i}}$ for some $p \in S$ or else that $\sup _{p \in S} \delta^{(i)}(p)=\sup _{k<w} m_{k}=m_{A}=$ $m_{A_{i}}$.

Corollary 5.2. If $A=\bigoplus_{i \in I} A_{i}$ where, for each $i \in I$ and each prime $p, A_{i}$ is a countable group such that the reduced part of the torsion subgroup of $A_{i}$ has an infinite p-primary component, then every free resolution (1) of $A$ splits over the $A_{i}$ 's.

Proof. Under these hypotheses, $m_{A_{i}}=\aleph_{0}$ and $T\left(A_{i}\right)=P$ for all $i$.

The stacked bases theorem can be interpreted as a theorem about free resolutions splitting over direct sums of cyclic groups. Indeed it is equivalent to the following: if $0 \rightarrow H \rightarrow G \rightarrow A \rightarrow 0$ is a free resolution of a direct sum $A$ of cyclic groups, then there exists a direct decomposition of $A$ into cyclic groups over which this resolution splits. Therefore, our next theorem can be viewed as a refined extension of the stacked bases theorem.

Theorem 5.3. If $A=\bigoplus_{i \in I} A_{i}$ where $\left|A_{i}\right|=\aleph_{0}$ for all $i$, then there is a direct decomposition $A=\bigoplus_{j \in J} B_{j}$ such that each $B_{j}$ is countable and each free resolution (1) of $A$ splits over the $B_{j}$ 's.

Although Theorem 5.3 can easily be derived from Theorem 5.1 by a simple combinatorial argument, we observe that this result, too, is a corollary of the following more comprehensive theorem. 
Theorem 5.4. Let $m$ be an infinite cardinal and suppose that $A=\bigoplus_{i \in I} A_{i}$ where $\left|A_{i}\right|<m$ for all $i \in I$. If

$$
0 \rightarrow H \rightarrow G \rightarrow A \rightarrow 0
$$

is a free resolution of $A$, then there is a direct decomposition $A=\bigoplus_{j \in J} B_{j}$ such that $\left|B_{j}\right|<m$ for all $j \in J$ and (1) splits over the $B_{j}$ 's. Moreover, if $m$ is uncountable, then we can choose the $B_{j}$ 's so that every free resolution of $A$ splits over the $B_{j}$ 's.

The proof of Theorem 5.4, however, depends on the following lemma.

Lemma 5.5. Let $m$ be an infinite cardinal and suppose that $A=\bigoplus_{i \in I} A_{i}$ where $\left|A_{i}\right|<m$ for each $i \in I$. Then $A=C \oplus A^{\prime}$ where $C$ is a direct sum of finite cyclic groups and $\operatorname{dim}(C / p C)=m$ for all primes $p$ for which $\operatorname{dim}\left(A_{t} / p A_{t}\right) \geq m$.

Proof. If $m=\aleph_{0}$, the lemma is trivial since we can then take $C=A$. Therefore we may assume that $m$ is uncountable. Notice also that it suffices to prove the lemma in the special case where $|I| \leq m$. Then, since there is nothing to prove if the set $S=\left\{p \in \mathbf{P}: \operatorname{dim}\left(A_{t} / p A_{t}\right)=m\right\}$ is empty, we may assume that $S \neq \varnothing$. The proof will be by induction on the cardinality of $I$.

We find it convenient to introduce certain special notation that will remain in effect throughout the proof. First, for each prime $p$, we introduce the additive function $\Gamma_{p}$ defined by $\Gamma_{p}(B)=B_{t} / p B_{t}$ for any abelian group $B$. If $J$ is a subset of $I$, then $A(J)=\bigoplus_{i \in J} A_{i}$. We let $\mu=\operatorname{cof}(|I|)$ and write $I=\bigcup_{\lambda<\mu} J_{\lambda}$ where $\left\{J_{\lambda}\right\}_{\lambda<\mu}$ is a continuous chain of subsets of $I$ with $\left|J_{\lambda}\right|<|I|$ for all $\lambda<\mu$. We also denote by $S^{*}$ that subset of $S$ consisting of all those primes $p$ such that $\operatorname{dim} \Gamma_{p}\left(A\left(J_{\lambda}\right)\right)=m$ for some $\lambda<\mu$. We shall consider several different case, always keeping in mind that the proof is by induction on $|I|$.

Case 1. $\operatorname{cof}(|I|)=w$ and $S=S^{*}$. Let $S_{n}=\left\{p \in S^{*}: \operatorname{dim} \Gamma_{p}\left(A\left(J_{n}\right)\right)=m\right\}$ and take $B_{0}=A\left(J_{0}\right)$. By the induction hypothesis, $B_{0}=C_{0} \oplus B_{0}^{\prime}$ where $C_{0}$ is a direct sum of finite cyclic groups with $\operatorname{dim}\left(C_{0} / p C_{0}\right)=m$ for all primes $p \in S_{0}$. Now for each $n \geq 1$, let $B_{n}=A\left(J_{n} \backslash J_{n-1}\right)$ and observe, by our induction hypothesis again, that $B_{n}=C_{n} \oplus B_{n}^{\prime}$ where $C_{n}$ is a direct sum of finite cyclic groups with $\operatorname{dim}\left(C_{n} / p C_{n}\right)=m$ for all $p \in S_{n} \backslash S_{n-1}$. Therefore $A=C \oplus A^{\prime}$ where $C=\bigoplus_{n<w} C_{n}$ and $A^{\prime}=\bigoplus_{n<w} B_{n}^{\prime}$. Since $S=S^{*}=$ $S_{0} \cup\left(S_{1} \backslash S_{0}\right) \cup \cdots \cup\left(S_{n} \backslash S_{n-1}\right) \cup \ldots$, clearly $\operatorname{dim}(C / p C)=m$ for all $p \in S$.

Case 2. $\operatorname{cof}(|I|)=w$ and $S \neq S^{*}$. Since, for each $p \in S \backslash S^{*}, \Gamma_{p}(A)$ is a vector space of dimension $m$ which is the union of the subspaces $\Gamma_{p}\left(A\left(J_{n}\right)\right)$, $n<w$, of smaller dimension, we must also have $\operatorname{cof}(m)=w$ in this case. But recalling that $m$ is uncountable, we see that there is a strictly increasing sequence

$$
m_{0}<m_{1}<m_{2}<\cdots<m_{n}<\cdots
$$

of uncountable regular cardinals with $m=\sup _{n<w} m_{n}$. We also list the elements of $S \backslash S^{*}$ as a finite or infinite sequence $p_{0}, p_{1}, \ldots, p_{k}, \ldots$ The proof, 
in this case, is based on the following fact, which derives from the theory of basic subgroups [5]: If $\operatorname{dim} \Gamma_{p}(B) \geq \kappa$ where $\kappa$ is an infinite cardinal with $\operatorname{cof}(\kappa) \neq \omega$, then $B=C \oplus B^{\prime}$ where $C$ is a direct sum of finite cyclic groups with $\operatorname{dim}(C / p C)=\kappa$. Indeed if $\operatorname{dim} \Gamma_{p}(B) \geq \kappa$ for all $p$ in some finite collection of primes, then we can choose $C$ so that $\operatorname{dim}(C / p C)=\kappa$ for all $p$ in that finite collection.

Recalling that, for $p \in S \backslash S^{*}, \Gamma_{p}(A)$ is the union of the subspaces $\Gamma_{p}\left(A\left(J_{n}\right)\right)$, each of dimension $<m$, we see that there is a subsequence $J_{0}^{\prime}, J_{1}^{\prime}, \ldots, J_{n}^{\prime}, \ldots$ of the $J_{n}$ 's such that $\operatorname{dim} \Gamma_{p}\left(A\left(J_{0}^{\prime}\right)\right) \geq m_{0}$ for $p=p_{0}$ and, for $n \geq 1$, $\operatorname{dim} \Gamma_{p}\left(A\left(J_{n}^{\prime} \backslash J_{n-1}^{\prime}\right)\right) \geq m_{n}$ for all $p=p_{k}$ with $k \leq n$. Now let $S_{n}^{\prime}=\{p \in$ $\left.S^{*}: \operatorname{dim} \Gamma_{p}\left(A\left(J_{n}^{\prime}\right)\right)=m\right\}, B_{0}=A\left(J_{0}^{\prime}\right)$ and, for $n \geq 1, B_{n}=A\left(J_{n}^{\prime} \backslash J_{n-1}^{\prime}\right)$. Applying both the induction hypothesis and the observation of the preceding paragraph, we obtain direct decompositions $B_{n}=C_{n} \oplus B_{n}^{\prime}$ where, for each $n<w, C_{n}$ is a direct sum of finite cyclic groups such that

$$
\operatorname{dim}\left(C_{n} / p C_{n}\right)= \begin{cases}m_{n} & \text { if } p=p_{k} \text { with } k \leq n, \\ m & \text { if } p \in S_{n}^{\prime} \backslash S_{n-1}^{\prime} .\end{cases}
$$

Clearly, $C=\bigoplus_{n<w} C_{n}$ is a direct summand of $A$ with the desired property that $\operatorname{dim}(C / p C)=m$ for all $p \in S$.

Case 3. $\mu=\operatorname{cof}(|I|)>w$. First observe that the assumption that $\operatorname{cof}(|I|) \neq w$ allows us to choose a subset $I^{*}$ of $I$ such that $\left|I^{*}\right|<|I|$ and $\operatorname{dim} \Gamma_{p}\left(A\left(I^{*}\right)\right)$ $=m$ for all $p \in S^{*}$; indeed we need only take $I^{*}=\bigcup_{p \in S^{*}} J_{\lambda(p)}$, where $\lambda(p)$ is the least ordinal $\lambda<\mu$ such that $\operatorname{dim} \Gamma_{p}\left(A\left(J_{\lambda}\right)\right)=m$. Then, by the induction hypothesis, $A\left(I^{*}\right)=C^{*} \oplus B$ where $C^{*}$ is a direct sum of finite cyclic groups with $\operatorname{dim}\left(C^{*} / p C^{*}\right)=m$ for all $p \in S^{*}$. If $S=S^{*}$, then $C=C^{*}$ is the desired direct summand of $A$. Thus we may assume that $S \neq S^{*}$. Then for any $p \in S \backslash S^{*}$, the $m$-dimensional vector space $\Gamma_{p}(A)$ is the union of the subspaces $\Gamma_{p}\left(A\left(J_{\lambda}\right)\right), \lambda<\mu$, where each of these subspaces has dimension strictly smaller than $m$. We shall find it convenient to consider two subcases.

First, suppose that $\mu<m$. Clearly, in this subcase, $m$ is a singular cardinal and, furthermore, there is a family $\left\{m_{\lambda}\right\}_{\lambda<\mu}$ of uncountable regular cardinals such that $m_{\lambda}<m$ for all $\lambda$ and $m=\sup _{\lambda<\mu} m_{\lambda}$. Under these circumstances, the index set $I$ will be the union of a continuous chain $\left\{I_{\lambda}\right\}_{\lambda<\mu}$ of subsets where $I_{0}=I^{*}$, each $I_{\lambda}$ is equal to some appropriate one of the original $J_{\lambda}$ 's, and $B_{\lambda}=A\left(I_{\lambda+1} \backslash I_{\lambda}\right)$ will contain a direct summand $C_{\lambda}$ which is a direct sum of finite cyclic groups with $\operatorname{dim}\left(C_{\lambda} / p C_{\lambda}\right)=m_{\lambda}$ for all $p \in S \backslash S^{*}$. First, we can certainly choose such $I_{\lambda}$ 's so that $\operatorname{dim} \Gamma_{p}\left(B_{\lambda}\right) \geq m_{\lambda}$ for all $p \in S \backslash S^{*}$. Then, from an earlier observation, this at least guarantees the existence of such a summand $C_{\lambda}$ with $\operatorname{dim}\left(C_{\lambda} / p C_{\lambda}\right)=m_{\lambda}$ for any given finite collection of $p$ 's in $S \backslash S^{*}$. But since $S \backslash S^{*}$ is at most countable and $\mu>w$, a further enlargement of the $I_{\lambda}$ 's will yield $C_{\lambda}$ 's with the requisite property for all $p \in S \backslash S^{*}$. Then $C=C^{*} \oplus\left(\bigoplus_{\lambda<\mu} C_{\lambda}\right)$ wil be the desired direct summand of $A$. 
Finally, suppose that $\mu=m$. (Recall that the possibility $\mu>m$ is excluded by the fact that $\mu=\operatorname{cof}(|I|) \leq|I| \leq m$.) This subcase is similar to but simpler than the preceding one. In fact, it suffices this time to choose the $I_{\lambda}$ 's so that $B_{\lambda}=A\left(I_{\lambda+1} \backslash I_{\lambda}\right)$ contains a direct summand $C_{\lambda}$ which is a direct sum of finite cyclic groups with $\operatorname{dim}\left(C_{\lambda} / p C_{\lambda}\right)=1$ for all $p \in S \backslash S^{*}$. This is clearly possible and, since $\mu=m, C=C^{*} \oplus\left(\bigoplus_{\lambda<\mu} C_{\lambda}\right)$ will once again be the desired direct summand of $A$.

We now turn to the proof of Theorem 5.4 and continue to employ the special notation used in the proof of Lemma 5.5. For $m=\aleph_{0}$, the theorem is equivalent to the stacked bases theorem. Thus we assume that $m$ is uncountable. Notice that the theorem is trivial if $I$ is finite or if $m_{A}<m$, and thus we assume that $I$ is infinite and $m_{A} \geq m$. But then, as in the proof of Theorem 5.1, $m_{A}=\sum_{i \in I} m_{A_{i}}$ where $m_{A_{i}}<m$ for all $i$. Since, however, any $A_{i}$ with $m_{A_{i}}=0$ can be neglected, we conclude that $m_{A}=\max \{m,|I|\}$. Next we reduce to the case where $|I| \leq m$.

Suppose that $m_{A}=|I|>m$. Notice that we may assume that $T(A)=\{p \in$ P: $\left.\operatorname{dim} \Gamma_{p}(A)=m_{A}\right\}$ is nonempty, since otherwise the conclusion of Theorem 5.4 follows immediately from Theorem 5.1. Because $\Gamma_{p}(A)=\bigoplus_{i \in I} \Gamma_{p}\left(A_{i}\right)$ with $\operatorname{dim} \Gamma_{p}\left(A_{i}\right)<m$ for all $i$, we must have $\left|\left\{i \in I: \Gamma_{p}\left(A_{i}\right) \neq 0\right\}\right|=m_{A}$ whenever $p \in T(A)$. We now claim that $I$ can be partitioned into a family of subsets $\left\{J_{\alpha}\right\}_{\alpha<m_{A}}$ where, for each $\alpha,\left|J_{\alpha}\right|=m$ and $\left|\left\{i \in J_{\alpha}: \Gamma_{p}\left(A_{i}\right) \neq 0\right\}\right|=m$ for all $p \in T(A)$. Indeed it is a routine transfinite induction to show that $I$ is the union of a continuous chain $\left\{I_{\alpha}\right\}_{\alpha<m_{A}}$ such that, for each $\alpha,\left|I_{\alpha}\right|=|\alpha| m$, $J_{\alpha}=I_{\alpha+1} \backslash I_{\alpha}$ has cardinality $m$ and $\left|\left\{i \in J_{\alpha}: \Gamma_{p}\left(A_{i}\right) \neq 0\right\}\right|=m$ for all $p \in T(A)$. If $B_{\alpha}=A\left(J_{\alpha}\right)$ for each $\alpha<m_{A}$, it will follow that $m_{B_{\alpha}}=m$ and $T\left(B_{\alpha}\right) \supseteq T(A)$ for all $\alpha$. Then $A=\bigoplus_{\alpha<m_{A}} B_{\alpha}$ and, by Theorem 5.1, any free resolution of $A$ splits over the $B_{\alpha}$ 's. But since each $B_{\alpha}$ is the direct sum of $m$ of the original $A_{i}$ 's we have indeed accomplished the reduction to the case $|I| \leq m=m_{A}$.

Continuing our proof of Theorem 5.4 under the current hypothesis that $m_{A}=$ $m \geq|I|$, we apply Lemma 5.5 and write $A=C \oplus A^{\prime}$ where $C$ is a direct sum of finite cyclic groups such that $\operatorname{dim}(C / p C)=m$ for all $p$ in $T(A)$. Recalling that $m$ is uncountable, we easily see that $C=\left(\bigoplus_{i \in I} K_{i}\right) \oplus C^{\prime}$ where, for each $i, K_{i} \oplus K_{i} \cong K_{i}$ and $\operatorname{dim}\left(K_{i} / p K_{i}\right)=\left|A_{i}\right| \aleph_{0}$ for all primes $p \in T(A)$. Now let $B_{i}=K_{i} \oplus A_{i}$ for all $i \in I$ and observe that

$$
A=C \oplus A^{\prime}=\left(\bigoplus_{i \in I} K_{i}\right) \oplus C^{\prime} \oplus A^{\prime} \cong\left(\bigoplus_{i \in I} K_{i}\right) \oplus A=\bigoplus_{i \in I} B_{i}
$$

Thus we may assume that $A=\bigoplus_{i \in I} B_{i}$, where clearly $\left|B_{i}\right|<m$ for each $i$, and furthermore

$$
\operatorname{dim} \Gamma_{p}\left(B_{i}\right)=\operatorname{dim}\left(K_{i} / p K_{i}\right)+\operatorname{dim} \Gamma_{p}\left(A_{i}\right)=\left|B_{i}\right|=m_{B_{i}}
$$


for all $p \in T(A)$. Therefore $T(A) \subseteq T\left(B_{i}\right)$ for each $i$ and Theorem 5.1 applies to show that every free resolution of $A$ splits over the $B_{i}$ 's. The proof of Theorem 5.4 is complete.

Lest we leave the reader with the general impression that free resolutions always decompose in some desirable fashion, we close with the following result.

Theorem 5.6. There exists a simply presented group $A$ and a free resolution (1) of $A$ which fails to split over any direct decomposition of $A$ into rank one subgroups.

Proof. We require some preliminary notation. If $p$ is a prime and $k$ is a positive integer, then $Z\left(p^{k}\right)$ denotes a cyclic group of order $p^{k}$. If $x$ is an element of the abelian group $A$, then $\|x\|$ will denote the height matrix of $x$. Thus $\|x\|$ is a doubly infinite matrix indexed by $\mathbf{P} \times w$ with the $(p, i)$ entry equal to the $p$-height in $A$ of $p^{i} x$. For each prime $p$, we take $A_{p}$ to be that abelian group with presentation in terms of generators $\left\{x_{i}^{(p)}\right\}_{i<w}$ subject only to the relations $p^{2 i+1} x_{i}^{(p)}=p^{2(i+1)} x_{i+1}^{(p)}$ for all $i<w$. Then the maximal torsion subgroup of $A_{p}$ is isomorphic to $\bigoplus_{i<w} Z\left(p^{2 i+1}\right)$ and $\left\|x_{0}^{(p)}\right\|=M_{p}$ where the entries $m_{q, i}$ of $M_{p}$ are given by

$$
m_{q, i}= \begin{cases}i & \text { if } q \neq p \\ 2 i & \text { if } q=p\end{cases}
$$

Then $A=\bigoplus_{p \in \mathrm{P}} A_{p}$ is a simply presented group and Theorem 4.2 implies that there is a free resolution (1) of $A$ such that $\delta(p)=\operatorname{dim}\left(\frac{H+p G}{p G}\right)$ is finite for every prime $p$. We claim that this resolution fails to split over every direct decomposition of $A$ into rank one summands. Indeed suppose $A=\bigoplus_{i<w} B_{i}$ where each $B_{i}$ is a subgroup of torsion free rank one. We shall show that each $T\left(B_{i}\right)$ consists of but a single prime and consequently (1) cannot split over the $B_{i}$ 's. In fact we shall demonstrate that the maximal torsion subgroup of each $B_{i}$ has exactly one infinite primary component. The crucial observation is that the analysis in [1] of the behavior of the Warfield invariants of $A$ relative to a decomposition of $A$ into rank one summands implies that to each $i<w$ there corresponds exactly one prime $p_{i}$ such that $B_{i}$ contains an element $y_{i}$ with the $p_{i}$-row of $\left\|y_{i}\right\|$ a sequence of successive even integers. In view of the structure of $A_{t}$ and the implication the foregoing observation has for the Ulm invariants of $B_{i}$ (see Lemma 22 in [6]), we conclude that $B_{i}$ contains all but a bounded part of the $p_{i}$-primary component of $A_{t}$. Since the $p_{i}$ 's necessarily exhaust $\mathbf{P}$, the $B_{i}$ 's have the property claimed above, namely, $T\left(B_{i}\right)=\left\{p_{i}\right\}$.

\section{REFERENCES}

1. D. Arnold, R. Hunter and E. Walker, Direct sums of cyclic valuated groups, Sympos. Math. 23 (1979), 77-84.

2. J. Cohen and H. Gluck, Stacked bases for modules over principal ideal domains, J. Algebra 14 (1970), 493-505. 
3. J. Erdös, Torsion-free factor groups of free abelian groups and a classification of torsion-free abelian groups, Publ. Math. Debrecen 5 (1957), 172-184.

4. L. Fuchs, Abelian groups, Publ. House Hungarian Acad. of Sci., Budapest, 1958.

5. __ Infinite Abelian groups, Vol. I, Academic Press, New York, 1970.

6. I. Kaplansky, Infinite Abelian groups, The University of Michigan Press, Ann Arbor, 1954.

Department of Mathematics, Baylor University, Waco, Texas 76798

Department of Mathematics, Vanderbilt University, Nashville, Tennessee 37235 